\title{
THE EFFECT OF EXPERIMENTALLY INDUCED HYPERVOLEMIA ON CARDIAC FUNCTION IN NORMAL SUBJECTS AND PATIENTS WITH MITRAL STENOSIS *
}

\author{
By TRUMAN G. SCHNABEL, JR., $\dagger$ HARALD ELIASCH, BENGT THOMASSON, \\ AND LARS WERKÖ $\ddagger$
}

(From the III Medical Service, St. Erik's Hospital, Stockholm, Sweden)

(Submitted for publication March 4, 1957 ; accepted September 11, 1958)

The physiologic differences between various types of laboratory preparations and unanesthetized man have been in part responsible for a wide diversion of opinion concerning the control of cardiac function in man (1). While numerous studies of the regulation of cardiac performance have been carried out in animals, relatively few studies have been done in $\operatorname{man}(2)$. The present work was undertaken in an attempt to clarify some of the mechanisms concerned with the control of cardiac function in man and has included studies in normal subjects and patients with mitral stenosis.

During a study of the relation existing between renal and cardiac function, rapid intravenous infusions were given to normal individuals and patients with various types of cardiac disorders (3). When the infusions consisted of isotonic solutions of dextran ${ }^{1}$ it was possible to analyze the relation of right heart filling pressure to cardiac output or right ventricular work, in a manner which closely simulated that used by Sarnoff and Berglund in the anesthetized dog (4), or Warren, Brannon, Weens and Stead in man (5). The present report is concerned with the effects on cardiac performance of varied degrees of expansion of the blood volume during 32 studies in 31 normal subjects and 16 studies in 15 patients with mitral stenosis.

\section{MATERIAL}

Normal Subjects. Twenty-seven normal volunteers who were either policemen, medical students, or nurses

* Presented in part at the World Congress of Physiology, Brussels, 1956. Aided by grants from the Swedish Medical Research Council and the Knut and Alice Wallenberg Foundation.

$\dagger$ Markle Scholar in Medical Science. Present address : Philadelphia General Hospital, Philadelphia 4, Penna.

$¥$ Present address: Sahlgrenska Sjukhuset, Goteborg, Sweden.

1 Macrodex $\mathrm{Rx}{ }^{(3)}$ of constant molecular size was generously supplied by Pharmacia Inc., Upsala, Sweden. were studied on an ambulatory basis, each resting for an hour before and after the study. On the basis of a history and physical examination none of these subjects showed evidence of cardiac or renal disease. In addition, four patients hospitalized because of cardiac symptoms but lacking objective findings of heart disease were studied. One of these subjects (No. 864) had high control values for oxygen consumption (339 and $358 \mathrm{ml}$. per minute), pulse rate (109 and 118 beats per minute) and cardiac index $\left(7.06\right.$ and $6.68 \mathrm{ml}$. per minute per $\mathrm{M}^{2}$ body surface area [B.S.A.]). The oxygen consumption, pulse rate and cardiac index remained high throughout the study. This subject did not give a history suggestive of hyperthyroidism and the renal excretion and thyroid uptake of $\mathrm{I}^{131}$ as well as the protein bound iodine were within normal limits.

One subject (No. 948 and 976) was studied on two occasions three and one-half months apart.

The age of the subjects ranged between 16 and 49 years. Nineteen subjects were male and 12 were female.

Patients. Seventeen patients with rheumatic heart disease and mitral stenosis were studied. The data in two patients have been excluded from the results. One of these patients developed an allergic reaction to dextran manifested by urticaria and a marked fall in blood pressure, while the other patient was unable to tolerate the procedure due to the severity of her cardiac disease. All of the patients had the classic signs of mitral stenosis. Most of the patients were only moderately limited by their heart disease and were classified functionally in group I or II according to the criteria of the American Heart Association. Two of the patients (Nos. 873 and 990) were more severely affected and were classified in group III. One of the patients was studied both before and after commissurotomy (No. 994 and 1009). This patient and one other (No. 979) had atrial fibrillation. All other patients had sinus rhythm. None of the patients were receiving cardiac medications at the time of the study except for three patients (Nos. 873, 994 and 1009) who were fully digitalized. The patients ranged in age from 26 to 49 years. Eleven patients were female and four were male.

Figure 1 shows the age and heart size of the patients as well as the physiologic data prior to the start of the intravenous infusions. The patients receiving infusions of dextran in isotonic saline solution had somewhat larger hearts, slightly lower cardiac indices and higher pulmonary arterial pressures than those receiving infusions of dextran in $\mathbf{5}$ per cent glucose and water. 

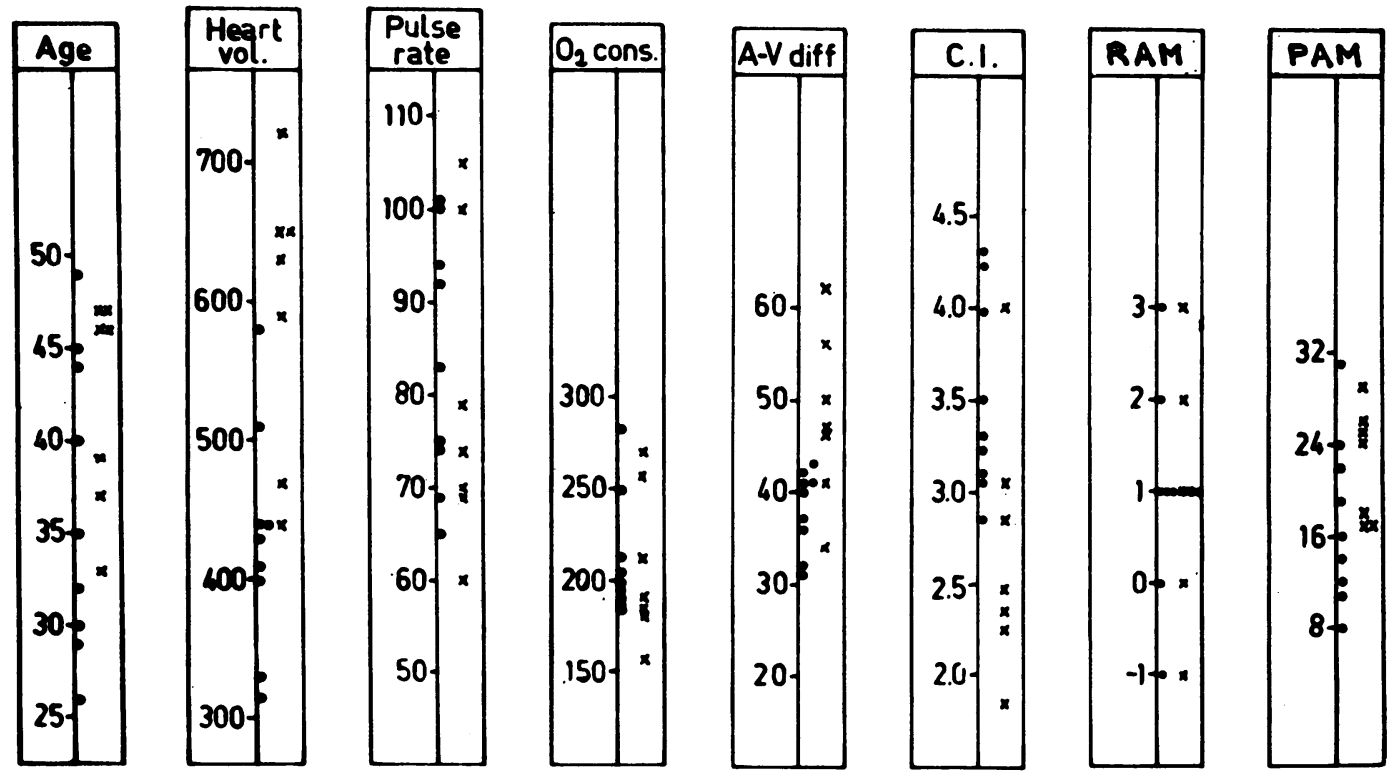

Fig. 1. Control Data Obtained on All Patients Receiving Infusions of Dextran in 5 Per Cent Glucose and Water (Black Dots) and Dextran in Isotonic Saline Solution (X)

Heart volume is given in ml. according to Liljestrand's method. RAM designates right atrial mean pressure in $\mathrm{mm}$. $\mathrm{Hg}$; PAM, pulmonary arterial mean pressure in $\mathrm{mm}$. $\mathrm{Hg} ; \mathrm{PR}$, pulse rate per minute; $\mathrm{O}_{2}$ cons., oxygen consumption in ml. per minute; CI, cardiac index in L. per minute per M. B.S.A.

\section{METHODS}

The pulmonary artery was catheterized according to the method of Cournand and Ranges (6-8). In four normal individuals (Nos. 855, 864, 907 and 926) and in three patients (Nos. 858, 863 and 925) a double lumen catheter was used. In two normal individuals (Nos. 867 and 986) and in five patients with mitral stenosis (Nos. 897, 967, 969, 979 and 994) two catheters were introduced, the tip of one catheter being placed in the pulmonary artery and the tip of the other in the right atrium. In all other studies the right atrial pressure was measured during passage of the catheter into the pulmonary artery, and again on withdrawal of the catheter just before the intravenous infusion was ended.

An indwelling arterial needle was placed in the brachial artery. Cardiac output was determined according to the direct Fick principle with simultaneous sampling of expired air and blood from the pulmonary and brachial arteries during a two minute period (9). The blood gases were determined in duplicate according to the technique of Van Slyke and Neill (10). Duplicate analyses of the expired air were carried out in a Haldane apparatus.

Blood samples for hemoglobin determinations were obtained periodically from the indwelling arterial needle. Hemoglobin content was measured from the amounts of oxyhemoglobin read in a Beckman spectrophotometer. The percentage decrease in hemoglobin was used as an indication of the increase in blood volume. Pressures were measured by Elema strain-gage manometers, the zero point of reference being $5 \mathrm{~cm}$. below the sternum at the level of the fourth costochondral junction.
The right ventricular work was calculated using the following formulae: Right ventricular work index (RVWI) in $\mathrm{Kg}$. per minute per $\mathrm{M}^{2}$ B.S.A. = cardiac index $X$ (mean pulmonary arterial pressure - mean right atrial pressure) $\times 13.6 / 1,000$.

Right ventricular stroke work index (RVSWI) in Gm.-M. per beat per M.' B.S.A. = stroke index $\times$ (mean pulmonary arterial pressure - mean right atrial pressure) $\times 13.6$.

Procedure. All studies were performed in the morning with the subject recumbent and in the postabsorptive state. Since the study included an evaluation of renal function and renal hemodynamics the patient was initially given a priming dose of inulin and para-aminohippurate intravenously. During the remainder of the study these substances were given at a constant rate with a motor driven syringe. As part of the renal study, collections of urine were begun 30 minutes following the priming dose of inulin and para-aminohippurate and were repeated every 10 to 15 minutes during the remainder of the procedure. Blood samples for the clearance determinations were obtained from the brachial artery at the time of each collection of urine.

Approximately one hour after the priming doses of inulin and para-aminohippurate had been given and 30 minutes after the catheter or catheters had been positioned, peripheral and pulmonary arterial blood samples were obtained with the collection of expired air and the measurement of right heart and peripheral arterial pressures. In most individuals two control determinations of the cardiac output were obtained one to two minutes apart. The mean of these two values was used for com- 
parison with the data obtained as the study progressed (9). Similar duplicate determinations of cardiac output were not made during the remainder of the study.

The infusion was begun immediately following the determination of the control values for oxygen consumption, arteriovenous oxygen difference, pulse rate and right heart and peripheral pressures.

Initial studies carried out with infusions of dextran alone proved unsatisfactory due to the occurrence of hemolysis. In two normal subjects (Nos. 851 and 855) and one patient (No. 858), a 10 per cent solution of dextran in water was used. In all subsequent studies dextran was infused as a 6 or 8 per cent solution with either 5 per cent glucose and water or isotonic saline solution. No evidence of hemolysis occurred with infusions of dextran solutions of this type. Thus, in 18 normal subjects and 15 patients with mitral stenosis, 6 per cent dextran was given either in 3 per cent glucose and water (13 normal subjects and nine patients with mitral stenosis) or in isotonic saline solution (five normal individuals and seven patients with mitral stenosis). In 12 normal subjects infusions of 5 per cent glucose and water (three subjects), isotonic saline solution (five subjects) and distilled water (four subjects) were given.

The infusions were given with a syringe driven by a motor at a constant rate so that approximately $25 \mathrm{ml}$. of fluid were injected per minute (11). Infusions of distilled water were given through a catheter, the tip of which lay in the right atrium. All other types of infusions were given through a polyethylene catheter which was inserted for a short distance into one of the antecubital veins. In normal subjects, solutions containing dextran were ordinarily given for 60 minutes. All other infusions in the normal individuals were given for periods of up to 100 minutes. In patients with mitral stenosis the infusions of dextran were terminated 30 to 50 minutes after their onset due to the rapid rise in pulmonary arterial pressure that ordinarily occurred at this time.

Cardiac output ordinarily was measured twice during the period of the infusion and again, in some instances, 15 to 20 minutes following the termination of the infusion. Measurement of right heart and peripheral pressures were carried out at 10 to 15 minute intervals at the onset of the infusion and more often during its later phases. All of the physiologic measurements were made without interrupting the infusion once it was begun.

No complications were noticed except in the two patients mentioned previously. None of the other subjects manifested signs of apprehension, dyspnea, orthopnea or pulmonary edema despite increases in blood volume of approximately 25 per cent and marked elevation of pulmonary arterial and right atrial pressures.

\section{RESULTS}

The necessity for infusing large amounts of water, glucose or saline with the dextran lead to a study of the cardiovascular effects of these fluids when they were given in a manner similar to that in which the dextran solutions were infused. Although infusions of distilled water produced hemolysis when given into a peripheral vein, no hemolysis developed when the infused water was given through a catheter, the tip of which lay in the right atrium.

In normal subjects infusions of isotonic saline solution, 3 per cent glucose in water and distilled water (Table I) produced a mean increase in blood volume of 5.3 per cent during the first 40 minutes of the infusion and 8.4 per cent when the infusion was continued for periods up to $80 \mathrm{~min}$ utes. Increases in blood volume of this magnitude produced no significant changes in heart rate, oxygen consumption, arteriovenous oxygen difference, cardiac index, stroke index, right atrial mean pressure and brachial arterial mean pressure (Table II). The mean value for pulmonary arterial mean pressure rose $2.5 \mathrm{~mm} . \mathrm{Hg}$ or 27 per cent $(0.05>p>0.02)$. Right ventricular stroke work index could be evaluated in only four studies rising less than 20 per cent in two subjects and 69 and 122 per cent in the other two individuals.

When dextran was given, with either 5 per cent glucose in water (Table III) or isotonic saline solution (Table IV), to normal subjects or patients without cardiovascular disease, the mean increase in blood volume was 12.9 per cent during the first 40 minutes of the infusion and 22.5 per cent when the infusion was continued for periods up to 60 minutes (Table V). A mean increase in blood volume of 12.9 per cent was not accompanied by significant changes in oxygen consumption, stroke index, brachial arterial mean pressure and the right ventricular stroke work index. Significant changes were found in the mean values for the arteriovenous oxygen difference $(-4.6 \mathrm{ml}$. per L. or -12 per cent, $\mathrm{p}<0.001)$, cardiac index $(+0.65 \mathrm{~L}$. per minute per M. ${ }^{2}$ B.S.A. or +17 per cent, $0.01>$ $\mathrm{p}>0.001)$, right atrial mean pressure $(+4.1$ mm. Hg or +168 per cent, $\mathrm{p}<0.001)$. The pulse rate increased four beats per minute or +5 per cent $(0.05>p>0.02)$.

During the latter period of the infusion when the mean increase in blood volume was 22.5 per cent, brachial arterial mean pressure was not significantly changed over control values. All other physiologic measurements were altered 
T. G. SCHNABEL, JR., H. ElIASCH, B. THOMASSON, AND L. WERKÖ

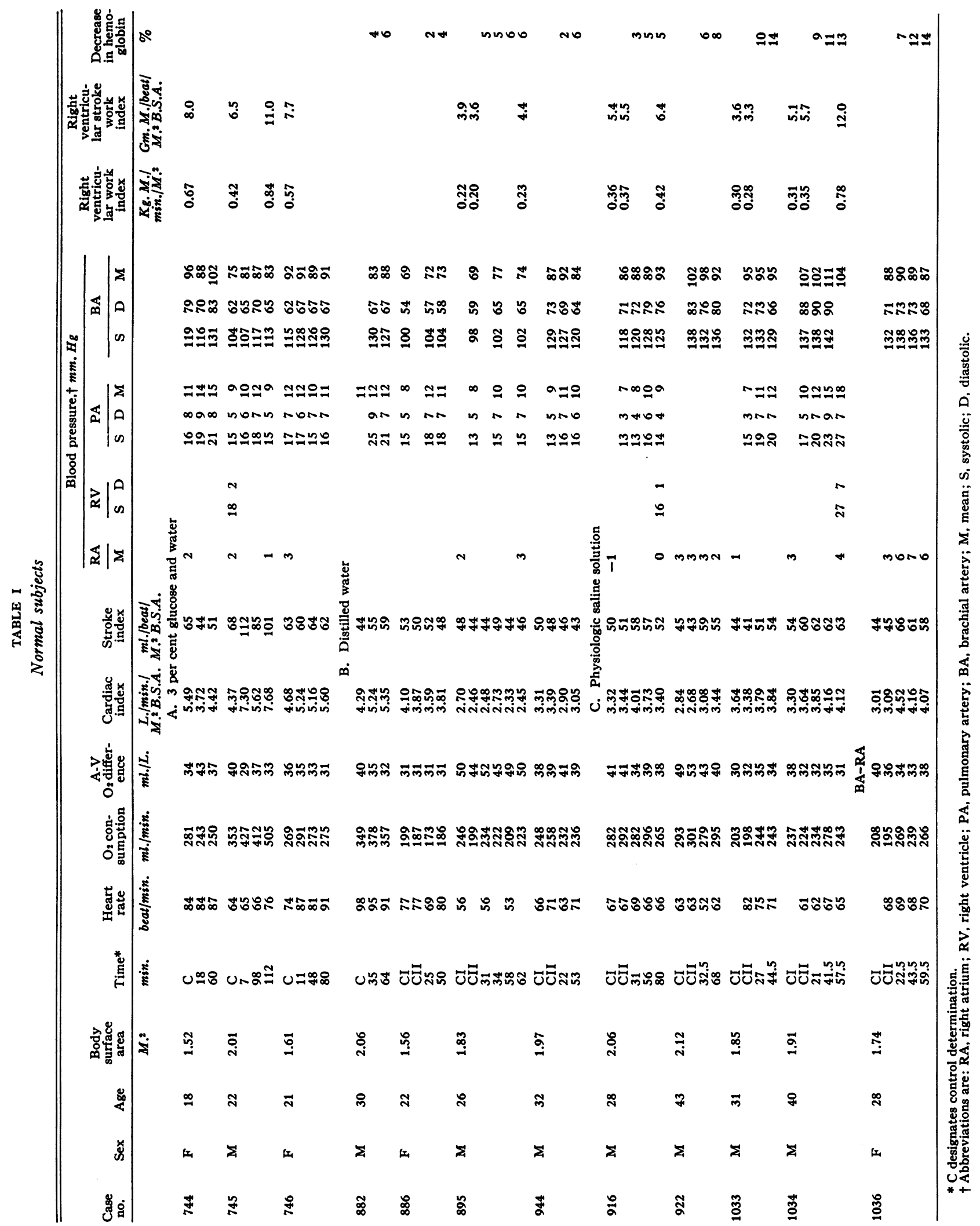


EFFECT OF INDUCED HYPERVOLEMIA ON CARDIAC FUNCTION

TABLE II

Normal subjects: $\mathrm{H}_{2} \mathrm{O}$, physiologic saline solution and 3 per cent glucose in $\mathrm{H}_{2} \mathrm{O}$

\begin{tabular}{|c|c|c|c|c|}
\hline & & \multirow[b]{2}{*}{ Control } & \multicolumn{2}{|c|}{$\begin{array}{c}\text { Difference from control values } \\
\text { during infusion }\end{array}$} \\
\hline & & & 20-39 min. & $39 \mathrm{~min}$. \\
\hline $\begin{array}{l}\text { Heart rate } \\
\quad(\text { beat } / \text { min. })\end{array}$ & $\begin{array}{l}\mathrm{N}^{*} \\
\mathrm{M} \\
\mathrm{SE} \\
\mathrm{SD} \\
\mathrm{p}\end{array}$ & $\begin{array}{c}12 \\
71.9 \\
3.39 \\
11.76\end{array}$ & $\begin{array}{c}9 \\
-3.4 \\
1.57 \\
4.72 \\
0.1>p>0.05\end{array}$ & $\begin{array}{l}12 \\
0 \\
1.49 \\
5.15 \\
0\end{array}$ \\
\hline $\begin{array}{l}\text { Oxygen consumption } \\
(\text { ml. } / \text { min. })\end{array}$ & $\begin{array}{l}N \\
M \\
S E \\
S D \\
p\end{array}$ & $\begin{array}{c}12 \\
261.6 \\
15.75 \\
54.57\end{array}$ & $\begin{array}{c}9 \\
17.9 \\
8.76 \\
26.27 \\
0.1>p>0.05\end{array}$ & $\begin{array}{c}12 \\
14.7 \\
7.66 \\
26.55 \\
0.1>p>0.05\end{array}$ \\
\hline $\begin{array}{l}\text { Arteriovenous oxygen difference } \\
\quad(m l . / L .)\end{array}$ & $\begin{array}{l}\mathrm{N} \\
\mathrm{M} \\
\mathrm{SE} \\
\mathrm{SD} \\
\mathrm{p}\end{array}$ & $\begin{array}{l}12 \\
38.6 \\
1.72 \\
5.96\end{array}$ & $\begin{array}{c}9 \\
-2.1 \\
1.43 \\
4.29 \\
0.2>p>0.1\end{array}$ & $\begin{array}{c}12 \\
-1.9 \\
1.28 \\
4.42 \\
0.2>p>0.1\end{array}$ \\
\hline $\begin{array}{l}\text { Cardiac index } \\
\quad\left(L . / \text { min./M. } .^{2} \text { B.S.A. }\right)\end{array}$ & $\begin{array}{l}\mathrm{N} \\
\mathrm{M} \\
\mathrm{SE} \\
\mathrm{SD} \\
\mathrm{p}\end{array}$ & $\begin{array}{l}12 \\
3.74 \\
0.245 \\
0.847\end{array}$ & $\begin{aligned} & 9 \\
& 0.36 \\
& 0.204 \\
& 0.613 \\
& 0.2>p>0.1\end{aligned}$ & $\begin{array}{c}12 \\
0.35 \\
0.197 \\
0.684 \\
0.2>p>0.1\end{array}$ \\
\hline $\begin{array}{l}\text { Stroke index } \\
\quad\left(m l . / \text { beat } / M .^{2} \text { B.S.A. }\right)\end{array}$ & $\begin{array}{l}\mathrm{N} \\
\mathrm{M} \\
\mathrm{SE} \\
\mathrm{SD} \\
\mathrm{p}\end{array}$ & $\begin{array}{l}12 \\
52.3 \\
2.57 \\
8.92\end{array}$ & $\begin{array}{c}9 \\
7.2 \\
2.54 \\
7.63 \\
0.05>p>0.02\end{array}$ & $\begin{array}{c}12 \\
4.6 \\
2.75 \\
9.51 \\
0.2>p>0.1\end{array}$ \\
\hline $\begin{array}{l}\text { Right auricular mean pressure } \\
(\mathrm{mm} . \mathrm{Hg})\end{array}$ & $\begin{array}{l}\mathrm{N} \\
\mathrm{M} \\
\mathrm{SE} \\
\mathrm{SD} \\
\mathrm{p}\end{array}$ & $\begin{array}{l}9 \\
2.0 \\
0.44 \\
1.32\end{array}$ & & $\begin{array}{ll} & 6 \\
& 0.6 \\
& 0.601 \\
& 1.5 \\
0.5 & >\mathrm{p}>0.3\end{array}$ \\
\hline $\begin{array}{l}\text { Pulmonary artery mean pressure } \\
\qquad(m m . H g)\end{array}$ & $\begin{array}{l}\mathrm{N} \\
\mathrm{M} \\
\mathrm{SE} \\
\mathrm{SD} \\
\mathrm{p}\end{array}$ & $\begin{array}{l}10 \\
9.2 \\
0.55 \\
1.75\end{array}$ & $\begin{array}{c}7 \\
2.3 \\
0.47 \\
1.25 \\
0.01>p>0.001\end{array}$ & $\begin{array}{c}10 \\
2.5 \\
0.95 \\
2.99 \\
0.05>\mathrm{p}>0.02\end{array}$ \\
\hline $\begin{array}{l}\text { Brachial artery mean pressure } \\
\quad(m m . H g)\end{array}$ & $\begin{array}{l}\mathrm{N} \\
\mathrm{M} \\
\mathrm{SE} \\
\mathrm{SD} \\
\mathrm{p}\end{array}$ & $\begin{array}{l}11 \\
87.8 \\
3.79 \\
12.58\end{array}$ & $\begin{array}{c}8 \\
1.4 \\
1.53 \\
4.34 \\
0.4>p>0.3\end{array}$ & $\begin{array}{c}11 \\
1.7 \\
1.74 \\
5.76 \\
0.4>p>0.3\end{array}$ \\
\hline $\begin{array}{l}\text { Right ventricular stroke work index } \\
\left(G m . M . / \text { beat } / M .^{2} \text { B.S.A.) }\right.\end{array}$ & $\begin{array}{l}\mathrm{N} \\
\mathrm{M} \\
\mathrm{SE} \\
\mathrm{SD} \\
\mathrm{p}\end{array}$ & $\begin{array}{l}7 \\
5.8 \\
0.66 \\
1.75\end{array}$ & & $\begin{array}{l}4 \\
1.6\end{array}$ \\
\hline $\begin{array}{l}\text { Decrease in hemoglobin } \\
(\%)\end{array}$ & $\begin{array}{l}N \\
M \\
S E \\
S D \\
p\end{array}$ & & $\begin{array}{l}9 \\
5.3\end{array}$ & $\begin{array}{l}9 \\
8.4\end{array}$ \\
\hline
\end{tabular}

* Abbreviations are as follows: $N$, number of observations; $M$, mean; SE, standard error ; SD, standard deviation ; p, $p$ value. 
EFFECT OF INDUCED HYPERVOLEMIA ON CARDIAC FUNCTION

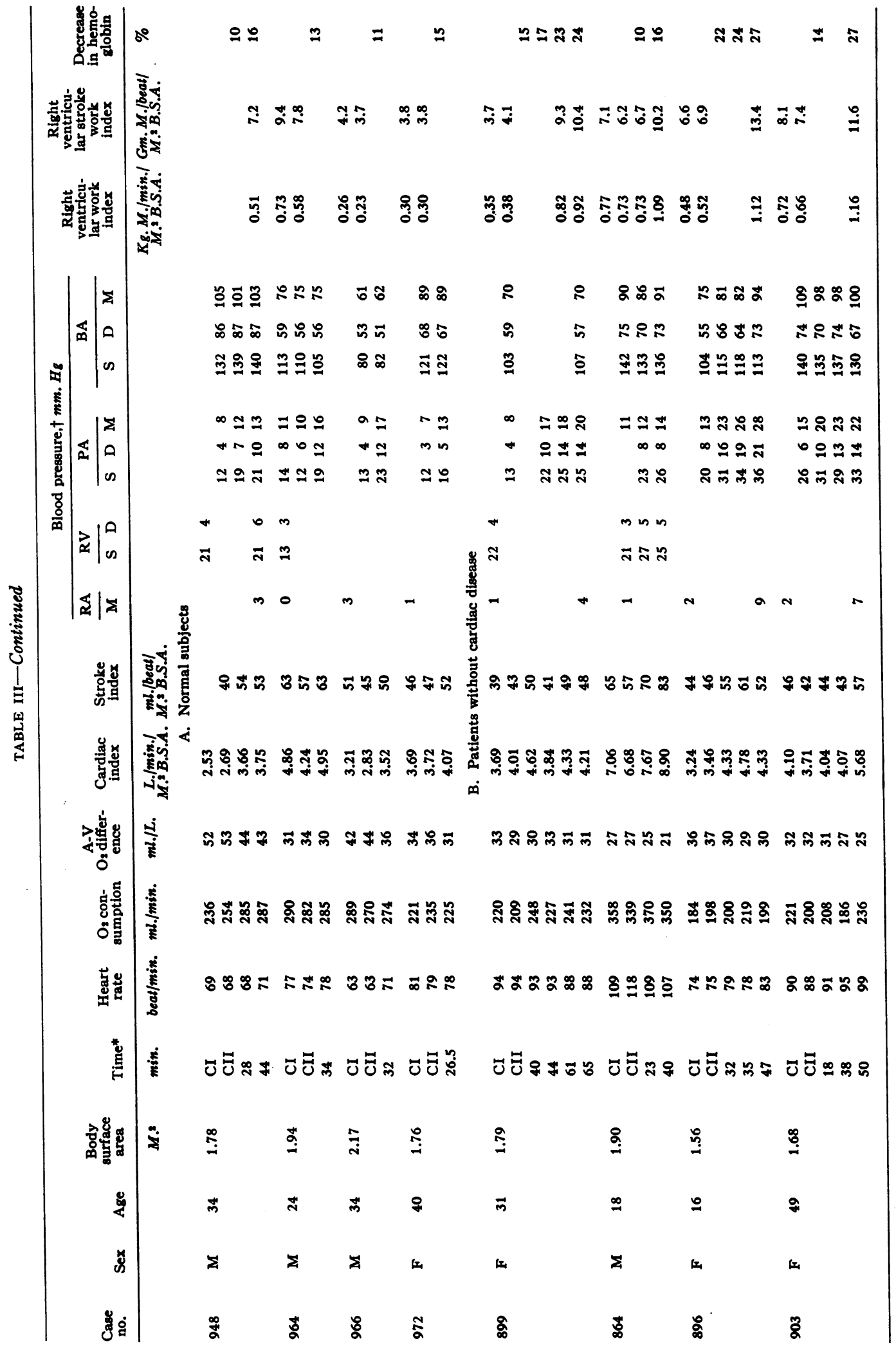


T. G. SCHNABEL, JR., H. ELIASCh, B. THOMASSON, AND L. WERKö

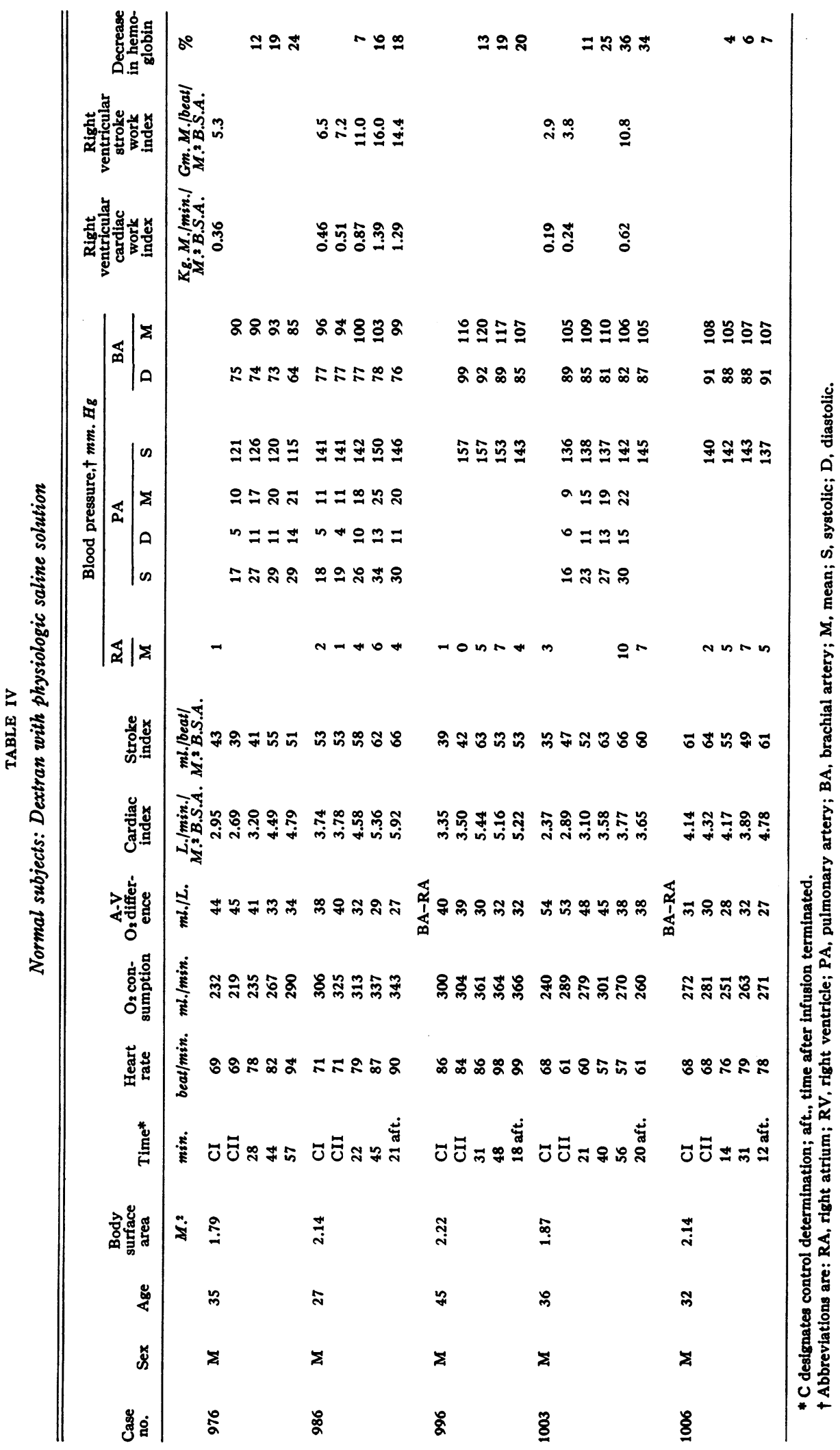


TABLE V

Normal subjects: Dextran in physiologic saline solution and dextran in 5 per cent glucose in $\mathrm{H}_{2} \mathrm{O}$

\begin{tabular}{|c|c|c|c|c|}
\hline & & \multirow[b]{2}{*}{ Control } & \multicolumn{2}{|c|}{$\begin{array}{c}\text { Difference from control values } \\
\text { during infusion }\end{array}$} \\
\hline & & & 20-39 min. & $39 \mathrm{~min}$. \\
\hline $\begin{array}{l}\text { Heart rate } \\
\quad(\text { beat } / \mathrm{min} .)\end{array}$ & $\begin{array}{l}\mathrm{N}^{*} \\
\mathrm{M} \\
\mathrm{SE} \\
\mathrm{SD} \\
\mathrm{p}\end{array}$ & $\begin{array}{l}20 \\
76.1 \\
2.87 \\
12.85\end{array}$ & $\begin{array}{c}17 \\
+4.0 \\
1.87 \\
7.73 \\
0.05>p>0.02\end{array}$ & $\begin{array}{c}16 \\
+7.3 \\
2.61 \\
10.46 \\
0.02>\mathrm{p}>0.01\end{array}$ \\
\hline $\begin{array}{l}\text { Oxygen consumption } \\
(\text { ml. } / \text { min. })\end{array}$ & $\begin{array}{l}\mathrm{N} \\
\mathrm{M} \\
\mathrm{SE} \\
\mathrm{SD} \\
\mathrm{p}\end{array}$ & $\begin{array}{c}20 \\
253.8 \\
9.68 \\
43.31\end{array}$ & $\begin{array}{c}17 \\
+7.1 \\
6.57 \\
27.08 \\
0.3>p>0.2\end{array}$ & $\begin{array}{c}16 \\
+18.7 \\
6.15 \\
24.62 \\
0.01>p>0.001\end{array}$ \\
\hline $\begin{array}{l}\text { Arteriovenous oxygen difference } \\
\quad(m l . / L .)\end{array}$ & $\begin{array}{l}\mathrm{N} \\
\mathrm{M} \\
\mathrm{SE} \\
\mathrm{SD} \\
\mathbf{p}\end{array}$ & $\begin{array}{l}20 \\
38.0 \\
1.73 \\
7.73\end{array}$ & $\begin{array}{c}17 \\
-4.6 \\
1.10 \\
4.54 \\
\mathrm{p}<0.001\end{array}$ & $\begin{array}{l}16 \\
-7.0 \\
1.38 \\
5.54 \\
p<0.001\end{array}$ \\
\hline $\begin{array}{l}\text { Cardiac index } \\
\quad\left(L . / \text { min./M. } .^{2} \text { B.S.A. }\right)\end{array}$ & $\begin{array}{l}\mathbf{N} \\
\mathrm{M} \\
\mathrm{SE} \\
\mathrm{SD} \\
\mathrm{p}\end{array}$ & $\begin{array}{l}20 \\
3.77 \\
0.207 \\
0.925\end{array}$ & $\begin{array}{c}17 \\
+0.65 \\
0.212 \\
0.875 \\
0.01>p>0.001\end{array}$ & $\begin{array}{c}16 \\
+1.17 \\
0.192 \\
0.769 \\
p<0.001\end{array}$ \\
\hline $\begin{array}{l}\text { Stroke index } \\
\quad\left(\text { ml. } / \text { beat } / M . .^{2} \text { B.S.A. }\right)\end{array}$ & $\begin{array}{l}\mathbf{N} \\
\mathbf{M} \\
\mathrm{SE} \\
\mathrm{SD} \\
\mathbf{p}\end{array}$ & $\begin{array}{l}20 \\
49.3 \\
1.90 \\
8.51\end{array}$ & $\begin{array}{c}17 \\
+6.6 \\
3.17 \\
13.08 \\
0.1>p>0.05\end{array}$ & $\begin{array}{c}16 \\
+10.0 \\
2.58 \\
10.30 \\
0.01>p>0.001\end{array}$ \\
\hline $\begin{array}{l}\text { Right atrial mean or right } \\
\text { ventricular indiastolic pressure } \\
(m m . H g)\end{array}$ & $\begin{array}{l}\mathrm{N} \\
\mathrm{M} \\
\mathrm{SE} \\
\mathrm{SD} \\
\mathrm{p}\end{array}$ & $\begin{array}{l}20 \\
2.3 \\
0.32 \\
1.45\end{array}$ & $\begin{array}{c}7 \\
+4.1 \\
0.86 \\
2.27 \\
0.01>p>0.001\end{array}$ & $\begin{array}{l}14 \\
+5.5 \\
0.73 \\
2.74 \\
p<0.001\end{array}$ \\
\hline $\begin{array}{l}\text { Pulmonary artery mean pressure } \\
(\mathrm{mm} . \mathrm{Hg})\end{array}$ & $\begin{array}{l}\mathrm{N} \\
\mathrm{M} \\
\mathrm{SE} \\
\mathrm{SD} \\
\mathbf{p}\end{array}$ & $\begin{array}{l}17 \\
10.4 \\
0.52 \\
2.15\end{array}$ & $\begin{array}{l}15 \\
+6.3 \\
0.85 \\
3.28 \\
\mathrm{p}<0.001\end{array}$ & $\begin{array}{c}14 \\
+9.9 \\
1.06 \\
3.97 \\
\mathrm{p}<0.001\end{array}$ \\
\hline $\begin{array}{l}\text { Brachial artery mean pressure } \\
\quad(m m . H g)\end{array}$ & $\begin{array}{l}\text { N } \\
\mathrm{M} \\
\mathrm{SE} \\
\mathrm{SD} \\
\mathbf{p}\end{array}$ & $\begin{array}{l}20 \\
89.1 \\
3.27 \\
14.62\end{array}$ & $\begin{array}{c}17 \\
+0.4 \\
1.04 \\
4.30 \\
0.8>p>0.7\end{array}$ & $\begin{array}{c}16 \\
+2.1 \\
1.56 \\
6.26 \\
0.2>p>0.1\end{array}$ \\
\hline $\begin{array}{l}\text { Right ventricular stroke work index } \\
\left(\text { Gm. M./beat } / M .^{2} B . S . A .\right)\end{array}$ & $\begin{array}{l}\mathrm{N} \\
\mathrm{M} \\
\mathrm{SE} \\
\mathrm{SD} \\
\mathbf{p}\end{array}$ & $\begin{array}{l}16 \\
5.6 \\
0.42 \\
1.69\end{array}$ & $\begin{array}{c}5 \\
+2.0 \\
0.91 \\
2.04 \\
0.1>p>0.05\end{array}$ & $\begin{array}{c}10 \\
+4.4 \\
0.89 \\
2.82 \\
\mathrm{p}<0.001\end{array}$ \\
\hline $\begin{array}{l}\text { Decrease in hemoglobin } \\
(\%)\end{array}$ & $\begin{array}{l}\mathrm{N} \\
\mathrm{M} \\
\mathrm{SE} \\
\mathrm{SD} \\
\mathrm{p}\end{array}$ & & $\begin{array}{c}17 \\
+12.9 \\
1.30 \\
5.35 \\
p<0.001\end{array}$ & $\begin{array}{c}16 \\
+22.5 \\
1.65 \\
6.61 \\
p<0.001\end{array}$ \\
\hline
\end{tabular}

* Abbreviations are as follows: $N$, number of observations; $M$, mean; $S E$, standard error; $S D$, standard deviation ; $p, p$ value. 
TABLE VI

Patients with mitral stenosis

\begin{tabular}{|c|c|c|c|c|c|c|c|}
\hline \multirow[b]{2}{*}{ Infused solution } & & \multicolumn{3}{|c|}{ Control } & \multicolumn{3}{|c|}{ 15-39 min. after the onset of infusions } \\
\hline & & $\begin{array}{l}\text { Dextran in } \\
\text { physiologic } \\
\text { saline } \\
\text { solution }\end{array}$ & $\begin{array}{l}\text { Dextran in } \\
5 \% \text { glucose } \\
\text { and water }\end{array}$ & $\begin{array}{l}\text { Combined } \\
\text { values }\end{array}$ & $\begin{array}{c}\text { Dextran in } \\
\text { physiologic } \\
\text { saline } \\
\text { solution }\end{array}$ & $\begin{array}{l}\text { Dextran in } \\
5 \% \text { glucose } \\
\text { and water }\end{array}$ & $\begin{array}{l}\text { Combined } \\
\text { values }\end{array}$ \\
\hline $\begin{array}{l}\text { Heart rate } \\
(\text { beat } / \min .)\end{array}$ & $\begin{array}{l}\mathrm{N}^{*} \\
\mathbf{M} \\
\text { SE } \\
\text { SD } \\
\text { p }\end{array}$ & $\begin{array}{c}7 \\
79.5 \\
6.32 \\
16.75\end{array}$ & $\begin{array}{c}9 \\
83.6 \\
4.52 \\
13.57\end{array}$ & $\begin{array}{l}16 \\
81.8 \\
3.66 \\
14.63\end{array}$ & $\begin{array}{l}7 \\
3.9 \\
2.224 \\
5.9 \\
0.2>p>0.1\end{array}$ & $\begin{array}{c}9 \\
.6 \\
2.16 \\
6.51 \\
0.8>p>0.7\end{array}$ & $\begin{array}{c}16 \\
1.72 \\
1.31 \\
6.25 \\
0.3>\mathrm{p}>0.2\end{array}$ \\
\hline $\begin{array}{l}\text { Oxygen consumption } \\
(\operatorname{ml} . / \min .)\end{array}$ & $\begin{array}{l}\mathbf{N} \\
\mathbf{M} \\
\mathrm{SE} \\
\mathrm{SD} \\
\mathbf{p}\end{array}$ & $\begin{array}{c}7 \\
208 \\
18.6 \\
45.58\end{array}$ & $\begin{array}{c}9 \\
211 \\
10.96 \\
32.87\end{array}$ & $\begin{array}{r}16 \\
209.8 \\
8.4 \\
33.6\end{array}$ & $\begin{array}{c}7 \\
16.4 \\
10.12 \\
26.82 \\
0.2>p>0.1\end{array}$ & $\begin{array}{c}9 \\
2.6 \\
6.77 \\
20.21 \\
0.8>\mathrm{p}>0.7\end{array}$ & $\begin{array}{c}16 \\
8.7 \\
6.295 \\
25.18 \\
0.2>0>0.1\end{array}$ \\
\hline $\begin{array}{l}\text { Arteriovenous oxygen } \\
\text { difference } \\
(m l . / L .)\end{array}$ & $\begin{array}{l}\mathbf{N} \\
\mathbf{M} \\
\mathrm{SE} \\
\mathrm{SD} \\
\mathbf{p}\end{array}$ & $\begin{array}{l}7 \\
48 \\
3.497 \\
9.266\end{array}$ & $\begin{array}{l}9 \\
38.11 \\
1.453 \\
4.36\end{array}$ & $\begin{array}{l}16 \\
42.44 \\
2.094 \\
8.376\end{array}$ & $\begin{array}{c}7 \\
-2.86 \\
2.26 \\
5.99 \\
0.3>p>0.2\end{array}$ & $\begin{array}{c}9 \\
-2.77 \\
.686 \\
2.059 \\
0.01>p>0.001\end{array}$ & $\begin{array}{c}16 \\
-2.81 \\
1.04 \\
4.81 \\
0.02>p>0.01\end{array}$ \\
\hline $\begin{array}{l}\text { Cardiac index } \\
(\text { L. } / \min . / M .2 \\
\text { B.S.A. })\end{array}$ & $\begin{array}{l}\mathbf{N} \\
\mathrm{M} \\
\mathrm{SE} \\
\mathrm{SD} \\
\mathrm{p}\end{array}$ & $\begin{array}{r}7 \\
2.707 \\
.256 \\
.678\end{array}$ & $\begin{array}{r}9 \\
3.489 \\
.183 \\
.549\end{array}$ & $\begin{array}{c}16 \\
3.147 \\
.1548 \\
.619\end{array}$ & 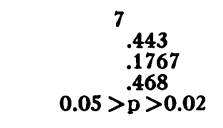 & $\begin{array}{c}9 \\
.3844 \\
.142 \\
.427 \\
0.05>\mathrm{p}>0.02\end{array}$ & $\begin{array}{c}16 \\
.410 \\
.1118 \\
.4475 \\
0.01>p>0.001\end{array}$ \\
\hline $\begin{array}{l}\text { Stroke index } \\
\quad(\text { ml./beat/M.2 B.S.A.) }\end{array}$ & $\begin{array}{l}\mathbf{N} \\
\mathbf{M} \\
\mathrm{SE} \\
\mathrm{SD} \\
\mathbf{p}\end{array}$ & $\begin{array}{r}7 \\
34.86 \\
2.99 \\
7.91\end{array}$ & $\begin{array}{l}9 \\
42.11 \\
1.148 \\
3.446\end{array}$ & $\begin{array}{r}16 \\
38.94 \\
1.68 \\
6.72\end{array}$ & $\begin{array}{c}7 \\
2.71 \\
1.491 \\
3.949 \\
0.2>\mathrm{p}>0.1\end{array}$ & $\begin{array}{l}9 \\
3 \\
1.365 \\
4.096 \\
0.1>\mathrm{p}>0.05\end{array}$ & $\begin{array}{c}16 \\
2.875 \\
.976 \\
3.903 \\
0.02>\mathrm{p}>0.01\end{array}$ \\
\hline $\begin{array}{l}\text { Right atrial mean or } \\
\text { right ventricular } \\
\text { indiastolic pressure } \\
\quad(\mathbf{m m} . \mathrm{Hg})\end{array}$ & $\begin{array}{l}\mathbf{N} \\
\mathrm{M} \\
\mathrm{SE} \\
\mathrm{SD} \\
\mathbf{p}\end{array}$ & $\begin{array}{l}7 \\
1 \\
.184 \\
.4873\end{array}$ & $\begin{array}{l}9 \\
2 \\
.842 \\
2.528\end{array}$ & $\begin{array}{l}16 \\
1.56 \\
.5 \\
2.00\end{array}$ & $\begin{array}{c}6 \\
3.83 \\
.749 \\
1.834 \\
0.01>p>0.001\end{array}$ & $\begin{array}{c}7 \\
4.7 \\
1.267 \\
3.356 \\
0.01>p>0.001\end{array}$ & $\begin{array}{l}13 \\
4.31 \\
.745 \\
2.69 \\
\text { p }<0.001\end{array}$ \\
\hline $\begin{array}{l}\text { Pulmonary artery } \\
\text { mean pressure } \\
(m m . H g)\end{array}$ & $\begin{array}{l}\mathbf{N} \\
\mathrm{M} \\
\mathrm{SE} \\
\mathrm{SD} \\
\mathbf{p}\end{array}$ & $\begin{array}{r}7 \\
22.29 \\
1.73 \\
4.59\end{array}$ & \begin{aligned} \multicolumn{1}{l}{9} \\
17.44 \\
2.43 \\
7.29\end{aligned} & $\begin{array}{l}16 \\
19.56 \\
1.658 \\
6.634\end{array}$ & $\begin{array}{c}7 \\
18.285 \\
1.267 \\
3.355 \\
\mathrm{p}<0.001\end{array}$ & $\begin{array}{l}9 \\
9.888 \\
1.173 \\
3.519 \\
\text { p }<0.001\end{array}$ & $\begin{array}{l}16 \\
13.56 \\
1.658 \\
5.442 \\
\text { p }<0.001\end{array}$ \\
\hline $\begin{array}{l}\text { Brachial artery } \\
\text { mean pressure } \\
(m m . H g)\end{array}$ & $\begin{array}{l}\mathbf{N} \\
\mathbf{M} \\
\mathrm{SE} \\
\mathbf{S D} \\
\mathbf{p}\end{array}$ & $\begin{array}{c}7 \\
100.5 \\
6.179 \\
16.368\end{array}$ & $\begin{array}{l}9 \\
75.33 \\
3.1175 \\
12.49\end{array}$ & $\begin{array}{l}16 \\
86.37 \\
4.75 \\
18.91\end{array}$ & $\begin{array}{c}7 \\
7.71 \\
2.356 \\
6.242 \\
0.02>p>0.01\end{array}$ & $\begin{array}{c}9 \\
1.89 \\
2.419 \\
7.258 \\
0.5>\text { p }>0.3\end{array}$ & $\begin{array}{c}16 \\
4.437 \\
1.821 \\
7.284 \\
0.05>\mathrm{p}>0.02\end{array}$ \\
\hline $\begin{array}{l}\text { Right ventricular } \\
\text { stroke work index } \\
(\mathrm{Gm} . M . / \text { beat/M.2 B.S.A.) }\end{array}$ & $\begin{array}{l}\text { N } \\
\mathrm{ME} \\
\mathrm{SD} \\
\mathrm{p}\end{array}$ & $\begin{array}{l}7 \\
9.73 \\
.6995 \\
1.853\end{array}$ & $\begin{array}{l}9 \\
8.9 \\
1.624 \\
4.872\end{array}$ & $\begin{array}{l}16 \\
9.26 \\
.960 \\
3.841\end{array}$ & $\begin{array}{l}\text { 6 } \\
7.45 \\
.818 \\
2.005 \\
\text { p }<0.001\end{array}$ & $\begin{array}{c}7 \\
4.086 \\
.7837 \\
2.076 \\
0.01>\mathrm{p}>0.001\end{array}$ & $\begin{array}{c}13 \\
5.638 \\
.770 \\
2.778 \\
\mathrm{p}<0.001\end{array}$ \\
\hline $\begin{array}{l}\text { Decrease in hemoglobin } \\
(\%)\end{array}$ & $\begin{array}{l}\mathbf{N} \\
\mathbf{M} \\
\mathrm{SE} \\
\mathrm{SD} \\
\mathrm{p}\end{array}$ & & & & $\begin{array}{c}7 \\
14.57 \\
1.97 \\
5.219 \\
\text { p }<0.001\end{array}$ & $\begin{array}{l}8 \\
18 \\
1.32 \\
3.742 \\
\mathrm{p}<0.001\end{array}$ & $\begin{array}{l}15 \\
16.28 \\
1.207 \\
4.671 \\
\mathrm{p}<0.001\end{array}$ \\
\hline
\end{tabular}

* Abbreviations are as follows: N, number of observations; $M$, mean; SE, standard error; SD, standard deviation; $p, p$ value.

significantly over the control values; pulse rate $(+7.3$ beats per minute or +9.6 per cent, $0.02>\mathrm{p}>0.01)$, oxygen consumption $(+18.7$ ml. per minute or +7 per cent, $0.01>\mathrm{p}>$ $0.001)$, arteriovenous oxygen difference $(-7 \mathrm{ml}$. per L. or -18 per cent, $\mathrm{p}<0.001)$, cardiac index $\left(+1.17\right.$ L. per minute per M. ${ }^{2}$ B.S.A. or + 31 per cent, $\mathrm{p}<0.001)$, stroke index $(+10 \mathrm{ml}$. per beat per M. ${ }^{2}$ B.S.A. or +20 per cent, $0.01>$ $\mathrm{p}>0.001)$, right atrial mean pressure $(+5.5$ $\mathrm{mm} . \mathrm{Hg}$ or +242 per cent, $\mathrm{p}<0.001)$, and right ventricular stroke work index $(+4.4 \mathrm{Gm} . \mathrm{M}$ per beat per M. ${ }^{2}$ B.S.A. or +78.6 per cent, $\mathrm{p}<$ 0.001 ).

During the control period, patients with mitral stenosis (Table VI) differed from normal subjects in having higher pulmonary arterial mean pressures (normals, $10.4 \mathrm{~mm} . \mathrm{Hg}$; patients, 19.5 $\mathrm{mm} . \mathrm{Hg}$ ) and greater right ventricular stroke work indices (normals, 5.6 Gm.M. per beat per M. ${ }^{2}$ B.S.A.; patients, $9.26 \mathrm{Gm} . \mathrm{M}$. per beat per M. ${ }^{2}$ B.S.A.).

Although patients with mitral stenosis receiving infusions of dextran in $\mathbf{5}$ per cent glucose and 
EFFECT OF INDUCED HYPERVOLEMIA ON CARDIAC FUNCTION

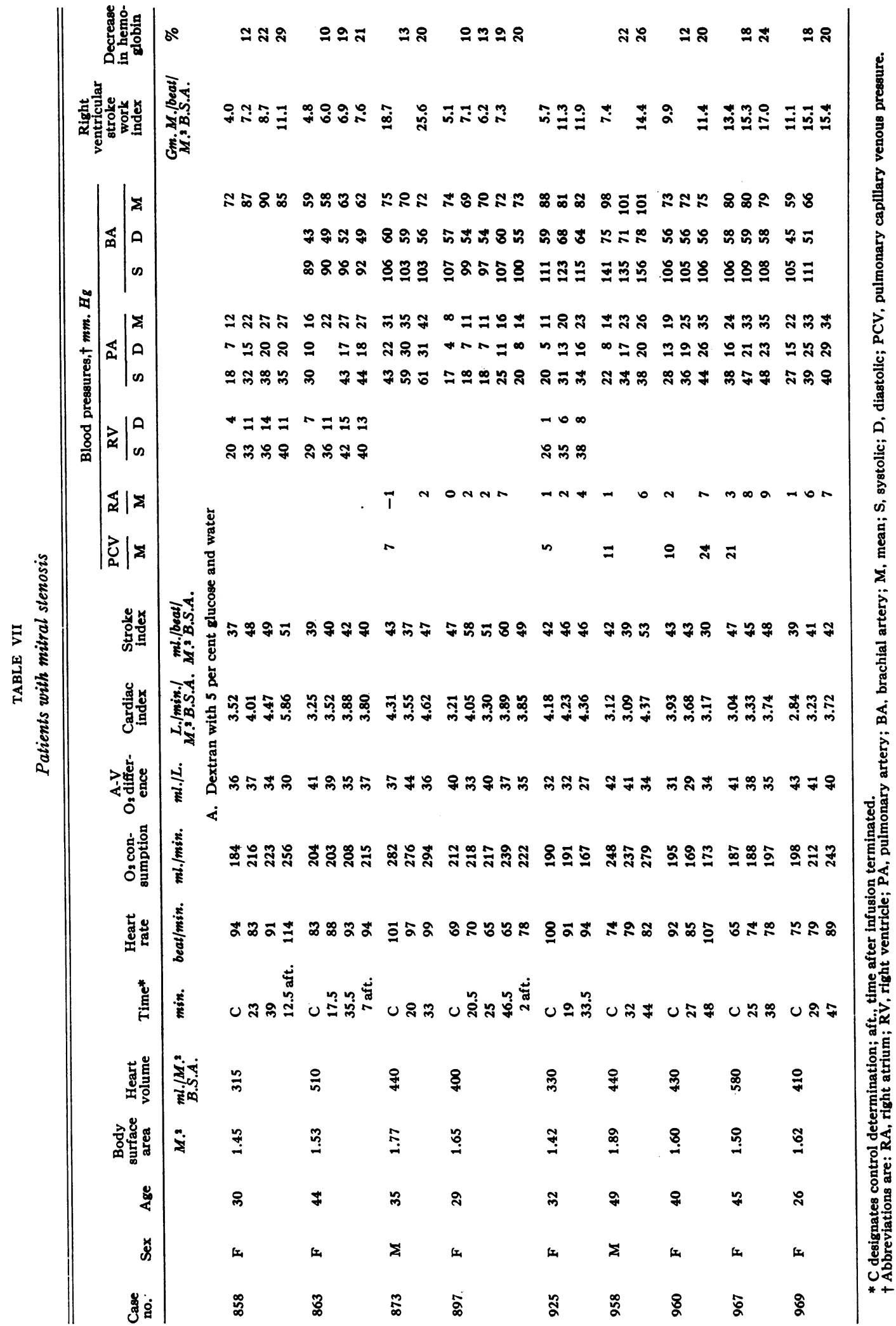


T. G. SCHNABEL, JR., H. ELIASCH, B. THOMASSON, AND L. WERKÖ

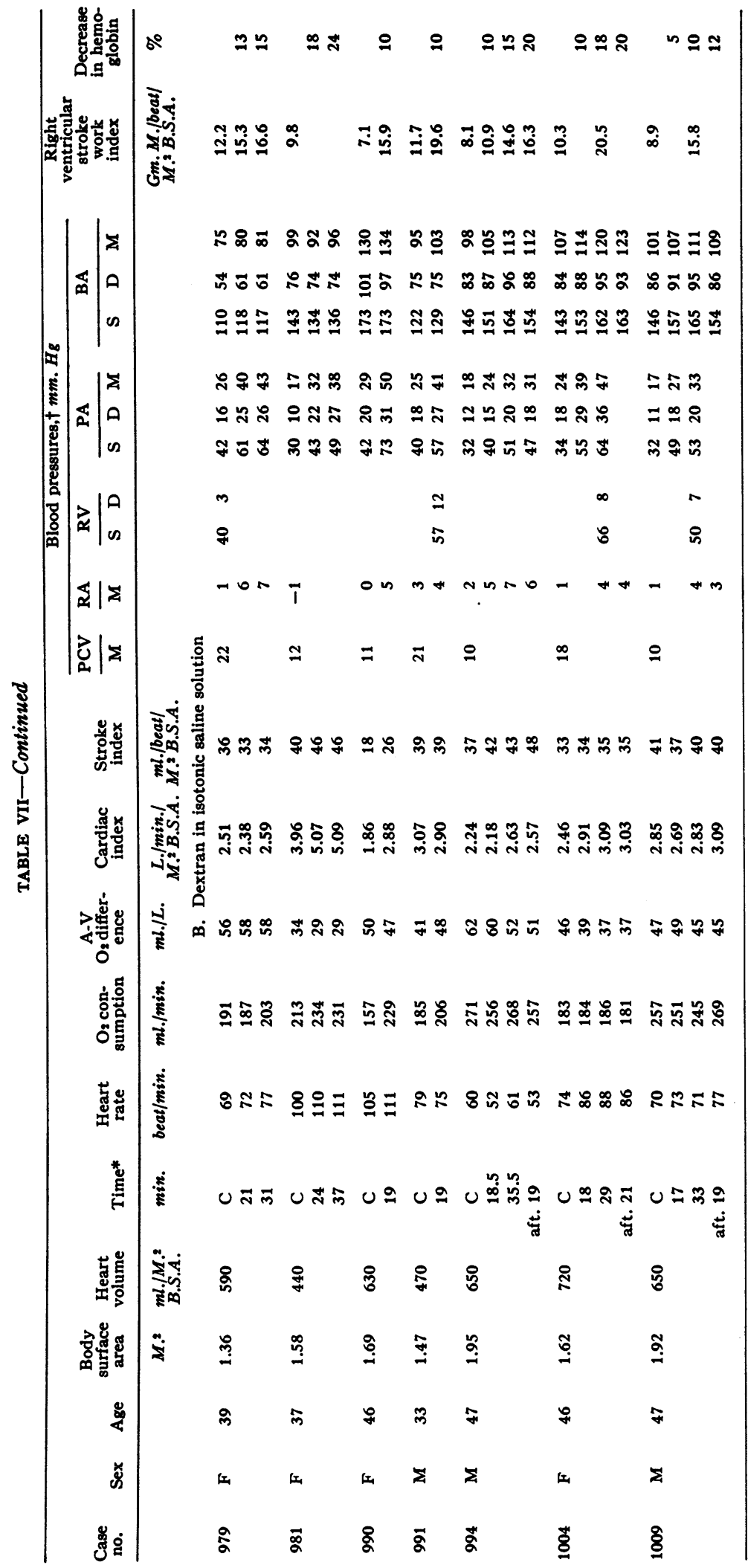


water (Table VII) showed only slight differences from the normal group in their mean control values for cardiac index, stroke index and arteriovenous oxygen difference, patients infused with dextran in isotonic saline solution (Table VII) had significantly lower cardiac and stroke indices and higher arteriovenous oxygen differences than the normal volunteers.

The results of infusions of dextran for periods of 40 minutes in patients with mitral stenosis and normal subjects were similar in producing slight but not significant changes in heart rate, oxygen consumption and brachial arterial pressure, while blood volume, cardiac and stroke indices and right heart filling pressure were increased by roughly comparable amounts.

The results differed in the greater increase in mean pulmonary arterial pressure (normals, + $6.3 \mathrm{~mm} . \mathrm{Hg}$; patients, $+13.6 \mathrm{~mm}$. $\mathrm{Hg}$ ) (Figure 2), and right ventricular stroke work index (normals, +2 Gm.M. per beat per M. ${ }^{2}$ B.S.A.; patients, + 5.6 Gm.M. per beat per M. ${ }^{2}$ B.S.A.) found in the patients. The difference was most marked in patients receiving infusions of dextran in isotonic saline solution in whom the mean increases in pulmonary arterial mean pressure and right ventricular stroke work index were $18 \mathrm{~mm}$. $\mathrm{Hg}$ and $7.45 \mathrm{Gm} . \mathrm{M}$. per beat per M. ${ }^{2}$ B.S.A., respectively, approximately three times that found in normal subjects.

While mean increases in right heart filling pressures of $5.5 \mathrm{~mm}$. $\mathrm{Hg}$ in normal subjects and 4.3 $\mathrm{mm}$. $\mathrm{Hg}$ in patients were associated with significant increases in the mean values for cardiac and stroke indices and right ventricular stroke work index, the coefficients of the regressions of right heart filling pressures to the change in these indices were not significant (Figures 3-5).

Examination of the data in individual studies shows instances in which significant changes in right heart filling pressures were associated with changes in cardiac output which could not be considered significant, as the standard error of a single determination of cardiac output in this laboratory has been demonstrated to be 7.2 per cent (9). A 10 per cent variability in duplicate determinations of cardiac output has been shown elsewhere $(12,13)$.

In seven normal volunteers cardiac indices were altered by less than 15 per cent while right

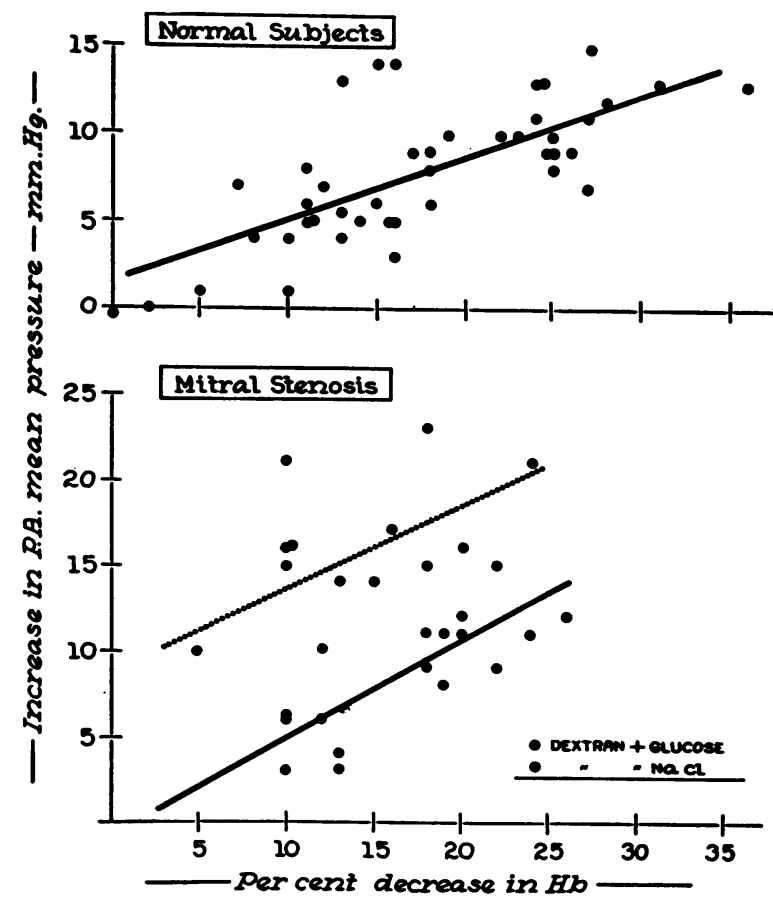

Fig. 2. Regressions For the Increase in PUlmonary Arterial Mean Pressure on the Percentage DeCrease in Hemoglobin

On the lower half of the figure separate regression lines are plotted for infusions of dextran in isotonic saline solution (dotted line) and dextran in 5 per cent glucose and water (solid line). The coefficients of the regression equations, $E=0.357 x+1.653$ (normal subjects) and $\mathrm{E}=0.576 \mathrm{x}-0.855$ (patients receiving infusions of dextran in 5 per cent glucose and water), are significant ( $p<$ 0.001 in each instance). The coefficient of the equation $\mathrm{E}=0.485 \mathrm{x}+9.035$ in patients infused with dextran in isotonic saline solution is not significant $(0.2>p>0.1)$.

heart filling pressures rose 4 to $12 \mathrm{~mm}$. $\mathrm{Hg}$. Stroke indices were unaltered or fell in five subjects in association with increases in right filling pressures of 5 to $10 \mathrm{~mm}$. Hg.

In six patients cardiac indices showed changes of less than 10 per cent while right heart filling pressures rose 2 to $6 \mathrm{~mm}$. $\mathrm{Hg}$. Stroke indices were unaltered or increased 1 to $2 \mathrm{ml}$. in three patients with increases in right heart filling pressures of 1 to $6 \mathrm{~mm}$. $\mathrm{Hg}$, while in three other patients stroke indices fell 1 to $10 \mathrm{ml}$. in association with increases in right heart filling pressures of 1 to $6 \mathrm{~mm}$. $\mathrm{Hg}$.

In all patients and in all normal subjects, save one, the right ventricular stroke work index rose in association with increases in right heart 


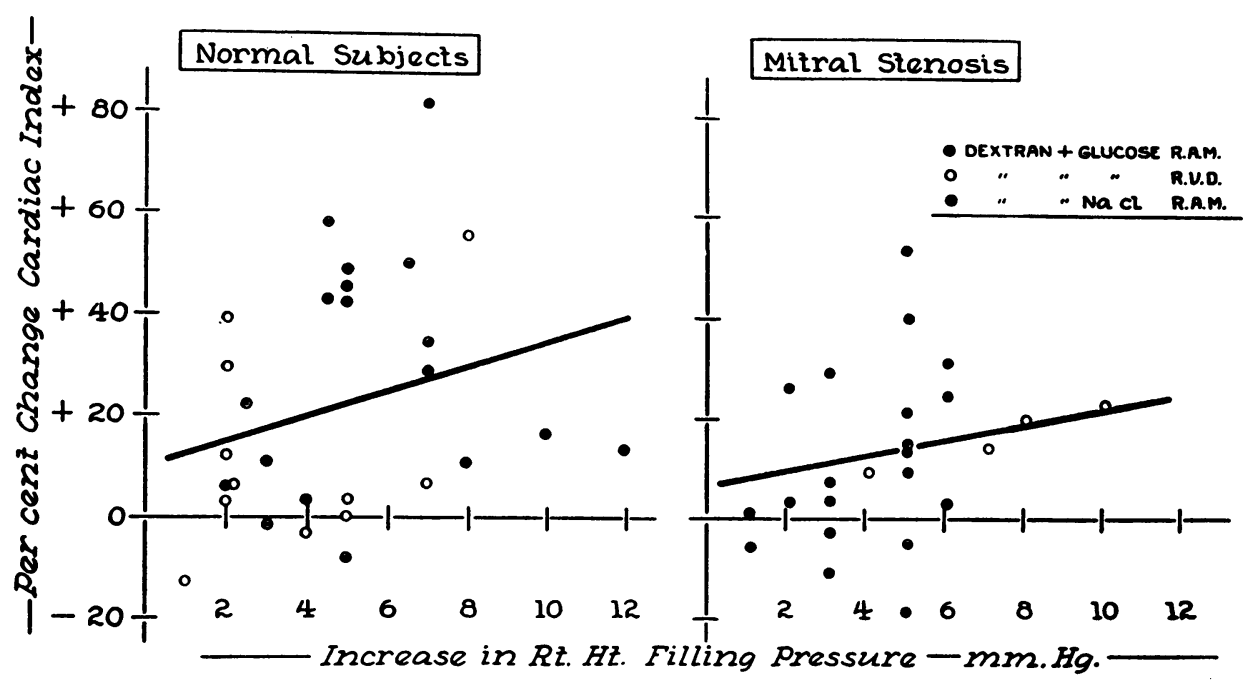

Fig. 3. Regressions for the Percentage Change in Cardiac Index on the Increase in Right Heart Filling Pressure

RAM designates right atrial mean pressure; RVD, right ventricular end-diastolic pressure. The coefficients of the regression equations, $E=2.53 x+9.974$ (normal subjects) and $E=$ $1.182 \mathrm{x}+6.406$ (patients with mitral stenosis), are not significant $(\mathrm{p}>0.1$ and $\mathrm{p}>0.3$, respectively).

filling pressures (Figure 6) and elevation of blood volume (Figure 7 ). The rise in right ventricular stroke work index was sometimes small in relation to the increase in right heart filling pres- sures being less than 40 per cent in five patients and two normal subjects with increases in right filling pressures of 2 to $8 \mathrm{~mm}$. $\mathrm{Hg}$ and 8 to $12 \mathrm{~mm}$. $\mathrm{Hg}$, respectively.

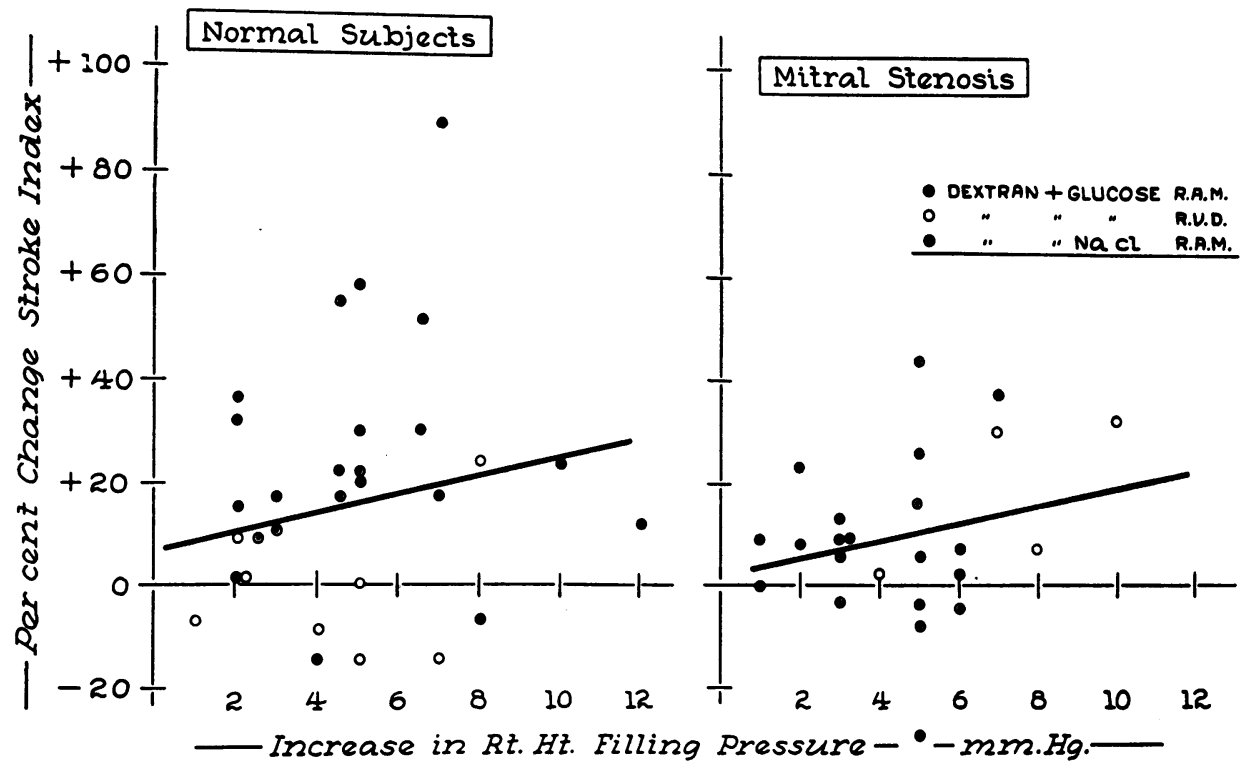

Fig. 4. Regressions for the Percentage Change in Stroke Index on the Increase in Right Heart Filling Pressures

RAM designates right atrial mean pressure; RVD, right ventricular end-diastolic pressure. The coefficients of the regression equations, $E=1.76 x+6.896$ (normal subjects) and $E=1.66 x$ +2.095 (patients with mitral stenosis), are not significant ( $p>0.3$ and $p>0.1$, respectively). 
A significant linear relation was found between the percentage decrease in hemoglobin in normal subjects and the percentage change in cardiac index $(0.02>p>0.01)$ (Figure 8), the percentage change in stroke index $(0.005>$ $p>0.001$ ) (Figure 9), and the change in right ventricular stroke work index $(0.05>\mathrm{p}>$ 0.02) (Figure 10). The correlation coefficients of $0.41,0.36$ and 0.47 relating the percentage decrease in hemoglobin in the normal subjects to these indices were also significant. In patients with mitral stenosis a significant linear relation was found between the percentage decrease in hemoglobin and the percentage change in cardiac index $(0.05>p>0.02)$. The correlation coefficient of 0.37 was also significant. No significant linear relation was found between the percentage decrease in hemoglobin in the patients with mitral stenosis and the percentage change in stroke index or the change in right ventricular stroke work index.

\section{DISCUSSION}

The effect of rapid infusions of albumin (5), dextran $(14)$ or saline $(15,16)$ on the cardiac output and pressures in the right atrium and

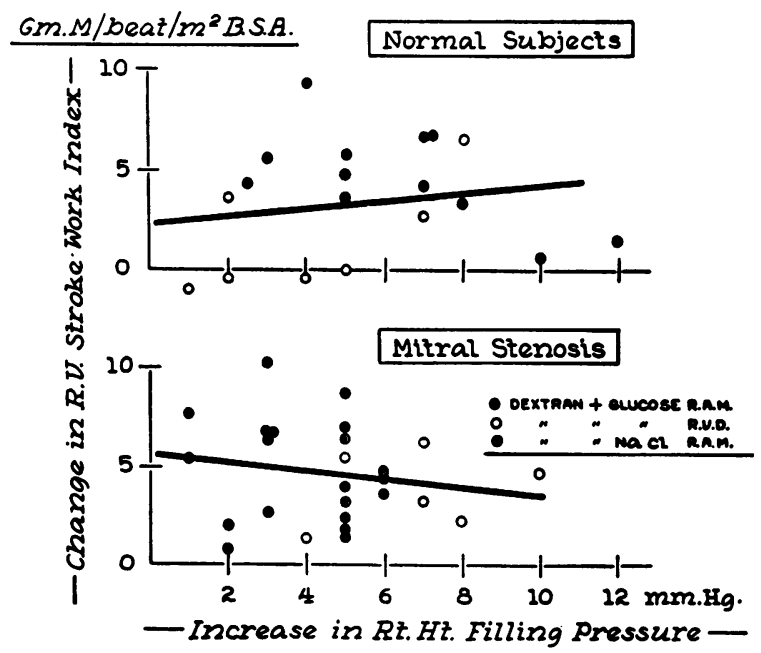

Fig. 5. Regressions for the Change in Right Ventricular (RV) Stroke Work Index on the InCREase in Right Heart Filling Pressure

RAM, right atrial mean pressure; RVD, right ventricular end-diastolic pressure. The coefficients of the regression equations, $E=0.1754 x+2.46$ (normal subjects) and $E=5.403-0.174 x$ are not significant $(p>0.3$ in each instance).

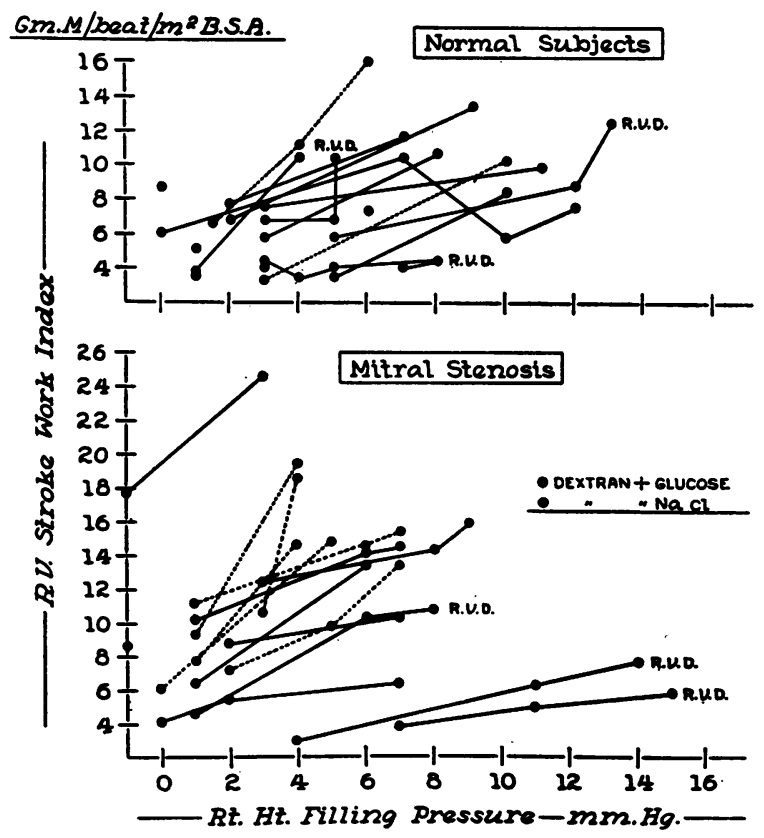

Fig. 6. The Relation Between Right Heart Filling Pressure and Right Ventricular Stroke Work Index in Each SubJect

The initial plots on the left hand side of the figure represent control values. All other plots occurred during the period of the infusions. Right atrial mean pressure was considered representative of right heart filling pressure except in those instances marked RVD, when right ventricular end-diastolic pressure was used.

pulmonary artery has been studied previously in man. While it has been found consistently that right atrial pressure rises as blood volume increases, the effect on cardiac output has varied, cardiac output remaining unchanged in one study (5) and rising in the others (14-16). This divergence in results may be due to variations in the magnitude of the blood volume increase, to physiologic differences among the subjects or to differences in the experimental procedures. In an effort to limit the effect of these variables, comparable studies were first carried out in normal volunteers and subsequently in patients with mitral stenosis.

In normal subjects infusions of 3 per cent glucose and water or isotonic saline solution increased blood volume 5 to 8 per cent with no significant change in right heart pressures and stroke or cardiac indices. Infusions of dextran in 5 per cent glucose and water or isotonic saline solution given in a similar manner to normal sub- 
Gm.M/beat $/ \mathrm{m}^{2}$ B.S.A.

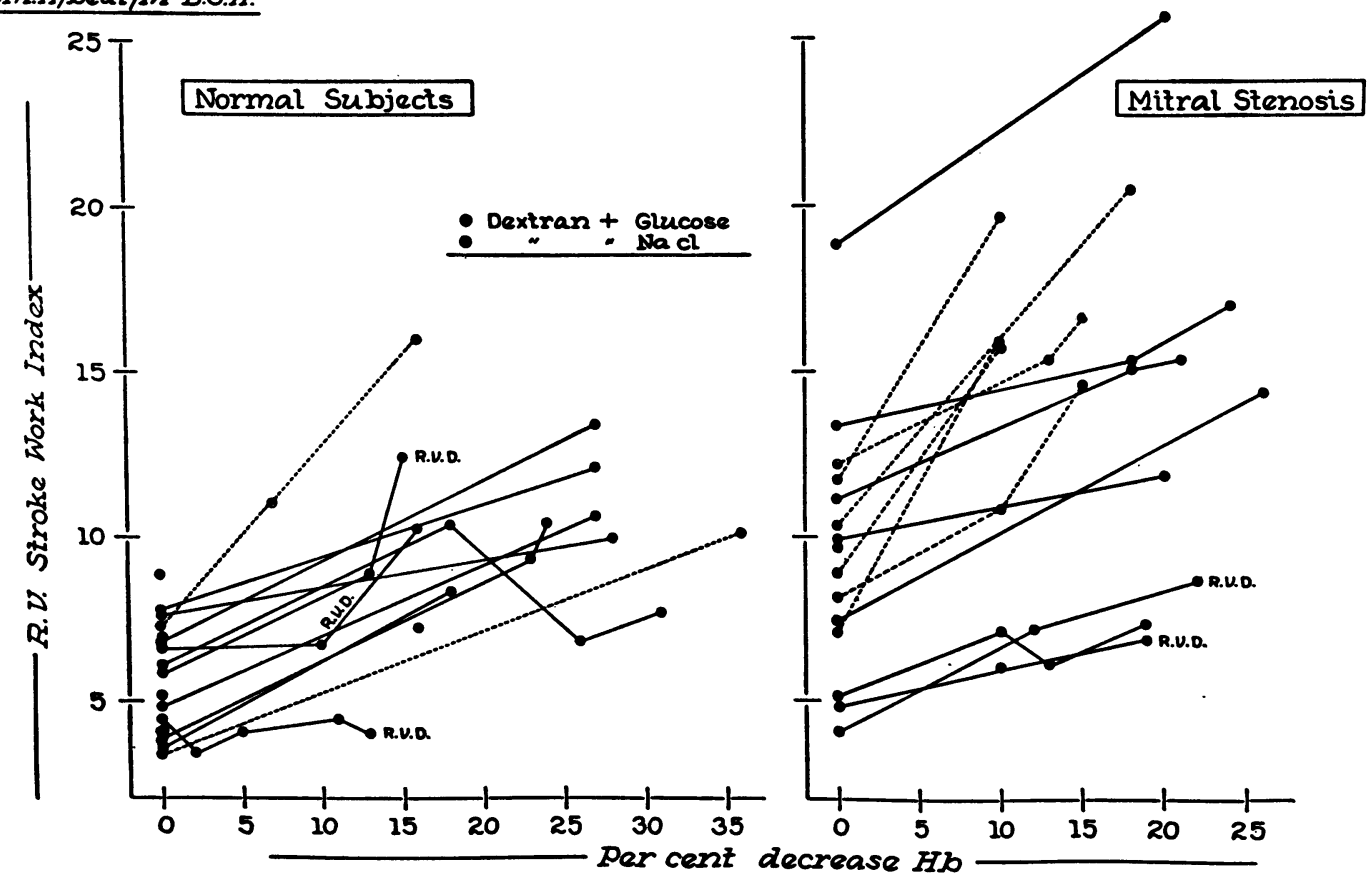

Fig. 7. The Relation Between the Percentage Decrease in Hemoglobin and Right Ventricular Stroke Work Index in Each Subject

The plots on the left of the graph are control values; subsequent plots were obtained during the period of the infusion. Right atrial mean pressure was used in calculating right ventricular stroke work index except in those instances marked RVD, when right ventricular end-diastolic pressure was used.

jects and patients with mitral stenosis increased blood volume 22 and 16 per cent, respectively, and were associated with significant increases in right heart and pulmonary arterial pressures, the increase in pressures being related to the degree of blood volume expansion, a relation that had been found previously in anesthetized dogs (17, 18).

Right heart filling pressure increased to a mean value of 6 to $7 \mathrm{~mm}$. $\mathrm{Hg}$, a level commonly found in patients with right heart failure, yet the absence of a fall in cardiac output either in the normal subjects or the patients with mitral stenosis suggests that the heart was not in a failing state.

The rise in pulmonary arterial pressure was significantly greater in patients with mitral stenosis, a finding which might be due either to alterations known to occur in the pulmonary vascular bed of these patients or perhaps to the presence of right ventricular hypertrophy.

In these studies the correct evaluation of the effects of alterations in right heart filling pres- sures on cardiac output and cardiac work is dependent on the relation existing between measured and effective pressure as well as the significance of right atrial mean pressure as an indicator of the filling pressure in the right ventricle.

Changes in intrathoracic and intrapericardial pressures were not measured during these studies making it impossible to determine the effective right heart pressures. Although the change in effective right atrial pressure was probably less than the measured pressure change, the difference was felt to be no greater than 1 to $2 \mathrm{~mm}$. $\mathrm{Hg}$.

In those instances in which right atrial mean and right ventricular end-diastolic pressures were measured simultaneously the latter was 1 to 4 $\mathrm{mm}$. $\mathrm{Hg}$ higher than the former during the control period. During the period of the infusion, right atrial mean pressure and right ventricular end-diastolic pressures rose in a comparable manner (Figure 11) as had been noted previously during exercise studies in patients with mitral stenosis (19). 
Besides increasing blood volume and elevating right heart filling pressure, an infusion of dextran causes other physiologic changes which in themselves may play some role in the regulation of cardiac output. Increased cardiac outputs found in dogs during dextran infusions were shown to be the result of a marked anemia and the associated decrease in the oxygen carrying capacity of the blood $(18,20)$. In the present studies the magnitude of the anemia was less than in the animal studies cited above, hematocrits being altered less than 30 per cent and hemoglobin levels never falling below $10 \mathrm{Gm}$., a level which has not been found to be associated with elevation of cardiac output in studies of patients with chronic anemia (21).

Cardiac output and right heart filling pressures may be altered by the occurrence of tricuspid insufficiency during dextran infusions in animals with open pericardiums (22). Examina-

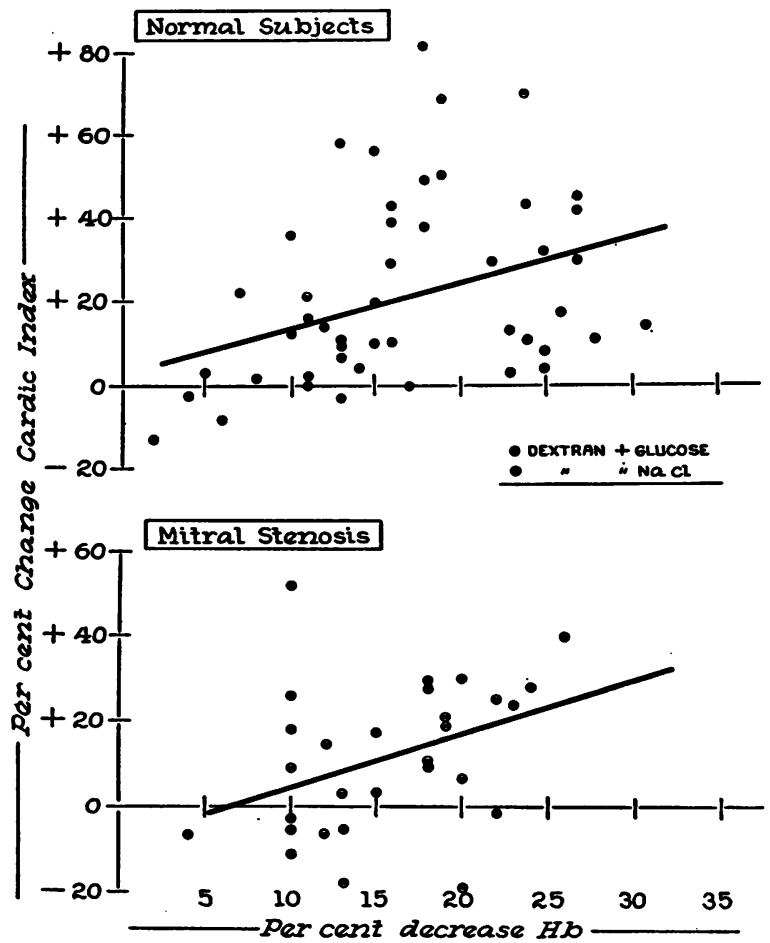

Fig. 8. Regressions for the Percentage Change in Cardiac Index on the Percentage Decrease in HEMOGLOBIN

The coefficient of the regression equations, $E=1.058 x$ +4.18 (normal subjects) and $E=1.188 x-6.78$ (patients with mitral stenosis), are significant $(0.02>\mathrm{p}>$ 0.01 and $0.05>p>0.02$, respectively).

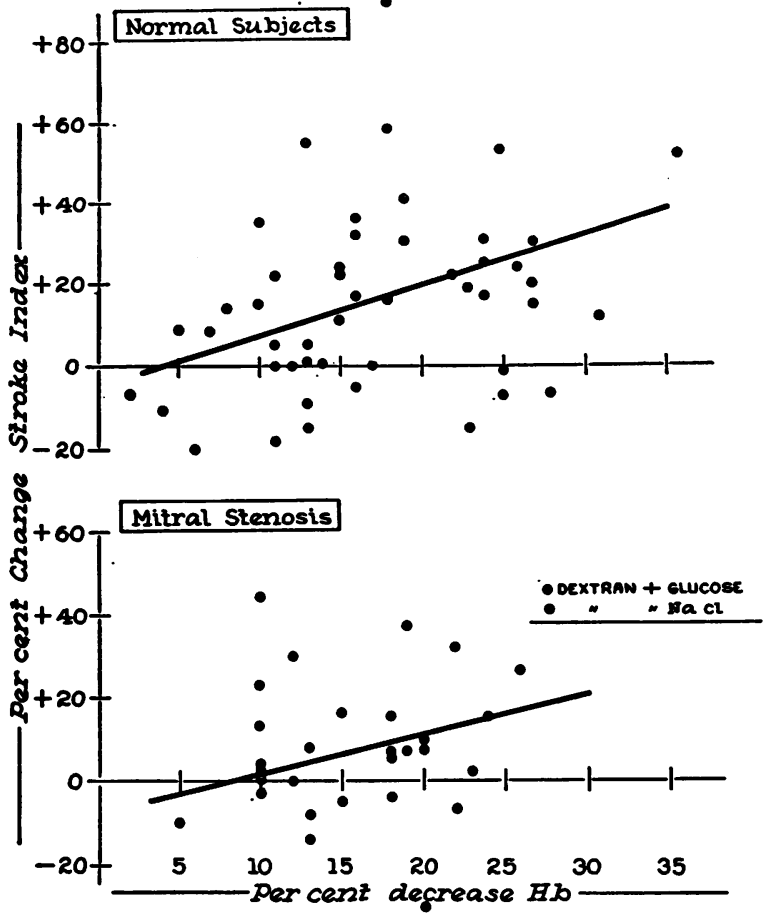

Fig. 9. Regressions for the Percentage Change in Stroke Index on the Percentage Decrease in HEMOGLOBIN

The coefficient of the regression equation, $E=1.2 \mathrm{x}-$ 4.95 in normal subjects, is significant $(p<0.005)$. The coefficient of the regression equation, $\mathrm{E}=0.9133 \mathrm{x}-6.78$ in patients with mitral stenosis, is not significant $(p>$ 0.1 ).

tions of the atrial pressure curves recorded at the end of the infusion periods failed to reveal evidence of tricuspid insufficiency in either normal volunteers or patients with mitral stenosis.

Data on renal blood flow obtained during the present studies show that infusions of dextran are often associated with changes in renal hemodynamics. In normal subjects renal blood flow was not significantly altered during infusions of isotonic saline solution or 5 per cent glucose and water. When normal volunteers received infusions of dextran with 5 per cent glucose and water or isotonic saline solution, renal blood flow increased, in some instances, to twice the control values. Increases in renal blood flow of this magnitude, although partially explicable on the basis of the measured change in cardiac output, would appear also to be indicative of vasodilitation in the renal vascular bed.

While the data in normal subjects and patients 


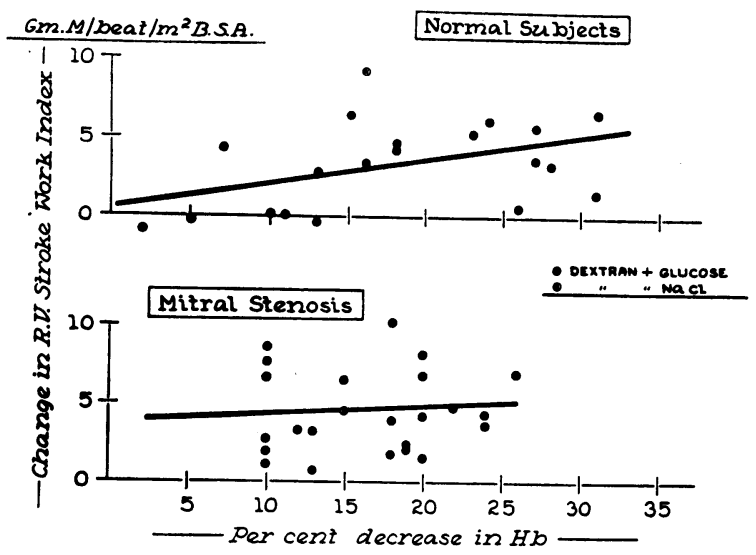

Fig. 10. Regressions for the Change in Right Ventricular Stroke Work Index on the Percentage DECREASE IN HEMOGLOBIN

The coefficient of the regression equation, $E=0.15 x+$ 0.656 in normal subjects, is significant $(0.05>p>0.02)$. The coefficient of the regression equation, $\mathrm{E}=0.044 \mathrm{x}+$ 3.829 in patients with mitral stenosis, is not significant $(\mathrm{p}>0.9)$.

with mitral stenosis show a mean rise in cardiac and stroke output occurring in association with increases in right heart filling pressures, no uniform way of relating these variables was found; indeed in 13 instances, changes in cardiac output of questionable significance occurred in the presence of increases in right heart filling pressures of 4 to $12 \mathrm{~mm}$. Hg. Previous studies in subjects with induced fluid retention have in some instances also failed to show a relation between right heart filling pressure and cardiac output (23).

Increases in right heart filling pressures of the magnitude produced in these studies are not usually encountered in the intact normal individual even under conditions of moderate to severe exertion (24) when the cardiac output is elevated to a far greater extent than was encountered in this work (25). The occurrence of only slight to moderate increases in cardiac output in association with abnormal increases in right heart filling pressures in healthy subjects implies the importance of other mechanisms by which cardiac output may be elevated from normal levels to the higher values associated with the physiologic stresses of daily life.

The significance of increases in right heart filling pressure to the elevation of cardiac output in patients with mitral stenosis cannot be clearly assessed since it is well known that these patients may be unable to increase their cardiac outputs to the extent found in normal subjects. It appears, however, that the effect on cardiac output of increases in right heart filling pressures, within the range studied, was essentially the same in healthy subjects and in patients with mitral stenosis.

Changes in blood volume were also associated with significant increases in the mean values of cardiac and stroke indices in both patients and normal subjects. The relation between blood volume changes and these indices was significant in normal subjects. In patients with mitral stenosis the relationship was significant only as regards the cardiac index. There were again eight normal subjects and five patients in whom maximum increases in blood volume of 10 to 31 per cent were associated with changes in cardiac output of less than 15 per cent, a change which should be considered of questionable significance in view of the limitations of the methods utilized.

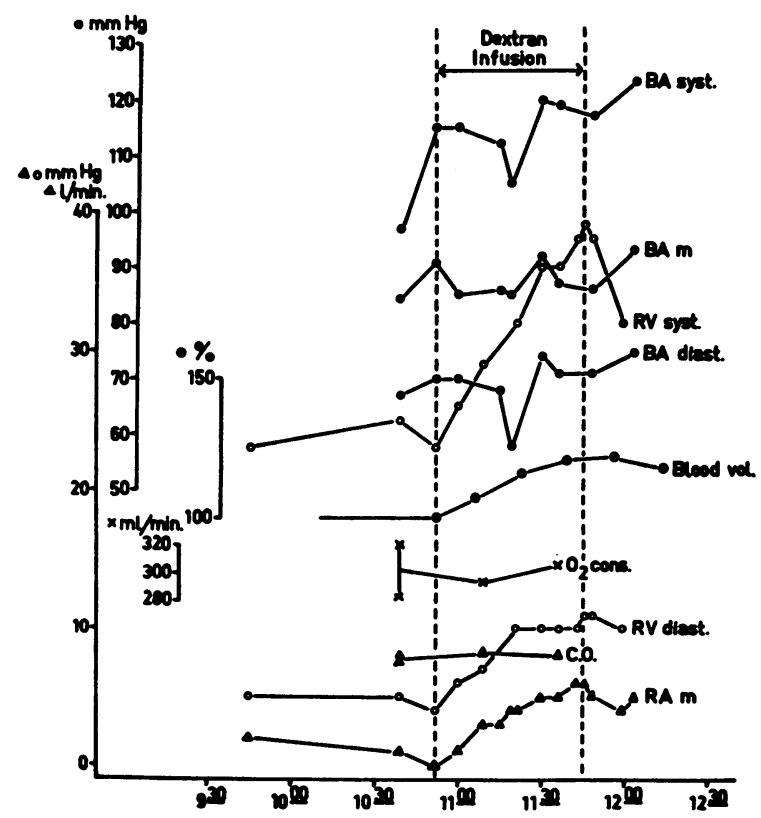

Fig. 11. Data Obtained During an Infusion of Dextran in 5 Per Cent Glucose and Water in a Normal Subject (No. 926)

The vertical dotted lines represent the start and the finish of the dextran infusion. Syst. designates systolic; Diast., diastolic; $M$, mean. 
The belief that right heart filling pressure is of dominant importance in the control of cardiac output in man has been based partly on studies conducted during pressure breathing (26), acute hemorrhage (27) and tilting $(28,29)$, experimental procedures which lead to a decreased venous return and a fall in the effective filling pressure of the right heart. Under these circumstances an ultimate fall in cardiac output would appear inevitable for the heart cannot pump out more blood than it receives. It does not necessarily follow that an increased venous return and an elevated cardiac filling pressure need be associated with an increased cardiac output.

The data in the present study suggest that the alterations in cardiac filling pressure and venous return cannot adequately explain the wide variations in cardiac output occurring in normal man; indeed the lack of a significant change in cardiac output in 13 subjects despite a steady increase in blood volume and right heart filling pressure suggests the presence of some mechanism by which cardiac output is maintained at a relatively constant level under these conditions.

In each subject, save one, increase in blood volume and elevation of right heart filling pressure were associated ultimately with an increase in right ventricular stroke work, a finding which is in accordance with Starling's law of the heart (30). However, no significant linear relation between these two variables and work was found except in the normal subjects where a significant linear relation existed between the percentage decrease in hemoglobin and the increase in right ventricular stroke work index. In individual subjects the increase in work for a given change in pressure was often far less than has been reported previously in animals. While this difference in the pressure volume relation (the ventricular function curve) of man and animal might be explained on the basis of the species studied, it might also be the result of the altered physiologic status of a laboratory preparation as compared to intact unanesthetized man. In a laboratory preparation, the normal state is sacrificed in varying degrees to reduce the number of variables under study to a minimum. In intact man these many variables operate simultaneously, each altering to some degree the manner in which the others would function were they to be operating alone.

Other investigators $(2,4)$ have felt that the response of the heart to a given filling pressure may vary widely as a result of changes presumably in myocardial tone or contractility ; functions of cardiac muscle which appear at least in part to be under neurohumoral control. The presence of these neurohumoral factors in intact man and their absence in many laboratory preparations would appear to be the most likely explanation of the disparity between the magnitude of the work increase found in this study and that found in the animal studies cited above.

The greatest increase in work for a given increase in blood volume or filling pressure was found in those patients with mitral stenosis whose hemodynamic findings showed the most marked deviations from normal. Whether this difference between patients and normal subjects was due to the presence of right ventricular hypertrophy, or the result of a change in myocardial contractility or tone initiated by some neurohumoral mechanism, cannot be determined from the present data.

The present study is an attempt to place in proper perspective the relation of increased blood volume and filling pressure to cardiac function in intact man. The data in no way deny to cardiac muscle its inherent ability of responding to increased tension with increased force (Starling's law of the heart), all other variables remaining constant. The data do, however, show that in normal man the response of cardiac muscle to an abnormal increase in filling pressure and blood volume is not of a sufficient degree to warrant the assumption that the filling pressure is necessarily the main determinant of the level of cardiac output or cardiac work. One explanation for the findings may lie in a continuous shift of cardiac function from one Starling curve to another as filling pressure rises. The implication is strong that a mechanism of this type or some other mechanism under neurohumoral control may be of more importance to the level of cardiac function than the small variations in filling pressure which occur in normal man or patients with mild to moderate forms of cardiac disease. 


\section{CONCLUSIONS}

1. Intravenous infusions of distilled water, 3 per cent glucose in water, physiologic saline solution and 6 or 8 per cent dextran in either 5 per cent glucose and water or physiologic saline solution were given at a constant rate of $25 \mathrm{ml}$. per minute for periods of 30 to 100 minutes to 31 normal subjects and 15 patients with mitral stenosis.

2. During infusions of water, physiologic saline solution and 3 per cent glucose in water in normal subjects, blood volume increased 5 to 8 per cent. No other significant changes in cardiovascular hemodynamics occurred.

3. Infusions of 6 or 8 per cent dextran solutions in normal subjects were associated with increases in blood volume of 20 to 35 per cent, rises in right heart filling pressures of 2 to $12 \mathrm{~mm}$. $\mathrm{Hg}$ and elevations of pulmonary arterial mean pressure of 3 to $15 \mathrm{~mm}$. $\mathrm{Hg}$. With the greatest expansion in blood volume, cardiac and stroke output were increased 31 and 20 per cent, respectively. In seven subjects, however, increases in right heart filling pressure of 4 to $12 \mathrm{~mm}$. $\mathrm{Hg}$ were associated with changes in cardiac output of less than 15 per cent.

4. In patients with mitral stenosis increases in blood volume of 10 to 29 per cent were associated with increases in right heart filling pressures of 1 to $10 \mathrm{~mm} . \mathrm{Hg}$ and increases in pulmonary arterial mean pressures of 8 to $23 \mathrm{~mm}$. $\mathrm{Hg}$. The mean increase in pulmonary arterial mean pressure of $13.56 \mathrm{~mm}$. $\mathrm{Hg}$ was twice that found in normal subjects. The increase in pulmonary arterial mean pressure was most marked in patients receiving infusions of 6 per cent dextran in physiologic saline solution, being on the average three times greater than the increase found in normal subjects. Mean values for cardiac and stroke output increased 13 and 7 per cent, respectively. In six patients cardiac output changed less than 10 per cent while right heart filling pressure increased 2 to $6 \mathrm{~mm}$. $\mathrm{Hg}$.

5. In all patients and in all normal subjects save one, maximum increases in blood volume and right heart filling pressure were associated with increases in right ventricular stroke work. In seven instances the increase in right ventricular work was relatively small compared to the increase in right heart filling pressure, the in- crease in right ventricular work being less than 40 per cent while right heart filling pressures rose 2 to $12 \mathrm{~mm}$. $\mathrm{Hg}$.

6. No significant relation appeared to exist in normal subjects or patients between the magnitude of the increase in right heart filling pressure and the percentage change in cardiac and stroke output or the change in right ventricular stroke work.

7. In normal subjects a significant linear relation was found between the degree of blood volume expansion and the percentage change in cardiac output, stroke output and the change in right ventricular stroke work. In patients with mitral stenosis a significant linear relation was found only between the degree of blood volume expansion and the percentage change in cardiac output.

8. The relatively small alterations in cardiac function occurring with the moderate to marked elevations of right heart filling pressure suggest the importance of factors other than filling pressure in the control of cardiac function in man.

\section{ACKNOWLEDGMENTS}

The technical assistance of Misses Elsa Ekberg, Lilly Backman, Annabrita Ohlson, Margareta Söderling, Ester Strandberg and Inga Landbrink is gratefully acknowledged.

\section{REFERENCES}

1. Symposium on the regulation of the performance of the heart. Physiol. Rev. 1955, 35, 90.

2. Rushmer, R. F. Applicability of Starling's law of the heart to intact, unanesthetized animals. Physiol. Rev. 1955, 35, 138.

3. Ek, J., Bucht, H., and Werkö, L. The influence of a rapid infusion of glucose on renal dynamics and sodium excretion in patients with arterial hypertension; preliminary report. Amer. Heart J. 1954, 48, 102.

4. Sarnoff, S. J., and Berglund E. Ventricular function. I. Starling's law of the heart studied by means of simultaneous right and left ventricular function curves in the dog. Circulation 1954, 9, 706.

5. Warren, J. V., Brannon, E. S., Weens, H. S., and Stead, E. A., Jr. Effect of increasing the blood volume and right atrial pressure on the circulation of normal subjects by intravenous infusions. Amer. J. Med. 1948, 4, 193.

6. Cournand, A., and Ranges, H. A. Catheterization of the right auricle in man. Proc. Soc. exp. Biol. (N. Y.) 1941, 46, 462.

7. Lagerlof, H., and Werkö, L. Studies on the circulation in man. A technique of venous catheterization with determination of cardiac output and simul- 
taneous recordings of the blood pressures, the electrocardiogram, phonocardiogram, and respiration. Acta med. scand. 1949, 132, 495.

8. Lagerlof, H., and Werkö, L. Studies on the circulation of blood in man. VI. The pulmonary capillary venous pressure pulse in man. Scand. J. clin. Lab. Invest. 1949, 7, 147.

9. Thomasson, B. Cardiac output in normal subjects under standard basal conditions. The repeatability of measurements by the Fick method. Scand. J. clin. Lab. Invest. 1957, 9, 365.

10. Van Slyke, D. D., and Neill, J. M. The determination of gases in blood and other solutions by vacuum extraction and manometric measurement. I. J. biol. Chem. 1924, 61, 523.

11. Ek, J. The influence of heavy hydration on the renal function in normal and hypertensive man. Scand. J. clin. Lab. Invest. 1955, 7, Suppl. 19.

12. Werkö, L. The influence of positive pressure breathing on the circulation in man. Acta med. scand. 1947, Suppl. 193.

13. Harvey, R. M., Ferrer, M. I., Cathcart, R. T., Richards, D. W., Jr., and Cournand, A. Some effects of digoxin upon the heart and circulation in man. Amer. J. Med. 1949, 7, 439.

14. Witham, A. C., Fleming, J. W., and Bloom, W. L. The effect of the intravenous administration of dextran on cardiac output and other circulatory dynamics. J. clin. Invest. 1951, 30, 897.

15. Doyle, J. T., Wilson, J. S., Estes, E. H., and Warren, J. V. The effect of intravenous infusions of physiologic saline solution on the pulmonary arterial and pulmonary capillary pressure in man. J. clin. Invest. 1951, 30, 345.

16. McMichael, J. Circulatory failure studied by means of venous catheterization. Advanc. intern. Med. 1947, 2, 64.

17. Henry, J. P., Gauer, O. H., and Sieker, H. O. The effect of moderate changes in blood volume on left and right atrial pressures. Circulat. Res. 1956, 4, 91.

18. Fowler, N. O., Franch, R. H., and Bloom, W. L. Hemodynamic effects of anemia with and without plasma volume expansion. Circulat. Res. 1956, 4, 319.

19. Eliasch, H. Pulmonary circulation at rest and on effort in mitral stenosis. Scand. J. clin. Lab. Invest. 1952, 4, Suppl. 4.
20. Sunahara, F. A., and Beck, L. Cardiovascular effects of acutely produced anemia in the normal dog. Amer. J. Physiol. 1954, 176, 139.

21. Brannon, E. S., Merrill, A. J., Warren, J. V., and Stead, E. A., Jr. The cardiac output in patients with chronic anemia as measured by the technique of right atrial catheterization. J. clin. Invest. $1945,24,332$.

22. Berglund, E., Sarnoff, S. J., and Isaacs, J. P. Ventricular function: Role of the pericardium in regulation of cardiovascular hemodynamics. Circulat. Res. 1955, 3, 133.

23. Albert, R. E., Smith, W. W., and Eichna, L. W. Hemodynamic changes associated with fluid retention induced in noncardiac subjects by corticotropin (ACTH) and cortisone; comparison with the hemodynamic changes of congestive heart failure. Circulation 1955, 12, 1047.

24. Holmgren, A. Circulatory changes during muscular work in man. with special reference to arterial and central venous pressures in the systemic circulation. Scand. J. clin. Lab. Invest. 1956, 8, Suppl. 24.

25. Asmussen, E., and Nielsen, M. Cardiac output during muscular work and its regulation. Physiol. Rev. 1955, 35, 778.

26. Cournand, A., Motley, H. L., Werkö, L., and Richards, D. W., Jr. Physiological studies of the effects of intermittent positive pressure breathing on cardiac output in man. Amer. J. Physiol. 1948, $152,162$.

27. McMichael, J., and Sharpey-Schafer, E. P. Cardiac output in man by direct Fick method. Effects of posture, venous pressure change, atropine, and adrenaline. Brit. Heart J. 1944, 6, 33.

28. Stead, E. A., Jr., Warren, J. V., Merrill, A. J., and Brannon, E. S. Cardiac output in male subjects as measured by the technique of right atrial catheterization. Normal values with observations on the effect of anxiety and tilting. J. clin. Invest. $1945,24,326$.

29. Hickam, J. B., and Pryor, W. W. Cardiac output in postural hypotension. J. clin. Invest. 1951, 30, 401.

30. Starling, E. H. The Linacre Lecture on the Law of the Heart. (Cambridge, 1915.) London, Longmans, Green and Co., 1918. 